\title{
Myeloperoxidase aggravates pulmonary arterial hypertension by activation of vascular Rho-kinase
}

\author{
Anna Klinke, ${ }^{1,2,3}$ Eva Berghausen,, ${ }^{1,2}$ Kai Friedrichs, ${ }^{1,2}$ Simon Molz, ${ }^{4}$ Denise Lau, ${ }^{4}$ Lisa Remane, ${ }^{1,2}$ \\ Matthias Berlin,, ${ }^{1,2}$ Charlotte Kaltwasser, ${ }^{1,2}$ Matti Adam,, Dennis Mehrkens, ${ }^{1,2}$ Martin Mollenhauer, ${ }^{1,2}$ \\ Kashish Manchanda, ${ }^{1,2}$ Thorben Ravekes, ${ }^{1,2}$ Gustavo A. Heresi, ${ }^{5}$ Metin Aytekin, ${ }^{6}$ Raed A. Dweik, ${ }^{5}$ \\ Jan K. Hennigs, ${ }^{7,8}$ Lukas Kubala,, ${ }^{3,9}$ Erik Michaëlsson, ${ }^{10}$ Stephan Rosenkranz,, ${ }^{1,2}$ Tanja K. Rudolph,, \\ Stanley L. Hazen, ${ }^{11}$ Hans Klose, ${ }^{8}$ Ralph T. Schermuly, ${ }^{12}$ Volker Rudolph, ${ }^{1,2}$ and Stephan Baldus ${ }^{1,2}$ \\ ${ }^{1}$ Heart Center, Department of Cardiology, ${ }^{2}$ Center for Molecular Medicine Cologne CMMC, University of Cologne, Cologne, \\ Germany. IInternational Clinical Research Center, Centre of Biomolecular and Cellular Engineering (CBCE), St. Anne's \\ University Hospital Brno, Brno, Czech Republic. ${ }^{4}$ University Heart Center Hamburg, University Medical Center Hamburg- \\ Eppendorf, Hamburg, Germany. ${ }^{5}$ Pulmonary and Critical Care Medicine, Respiratory Institute, and ${ }^{6}$ Pathobiology, Lerner \\ Research Institute, Cleveland Clinic, Cleveland, Ohio, USA. ${ }^{7}$ Cardiovascular Institute, Stanford University, School of Medicine, \\ Stanford, California, USA. ${ }^{8}$ Department of Pneumology, University Medical Center Hamburg-Eppendorf, Hamburg, Cermany. \\ ${ }^{9}$ Institute of Biophysics, Czech Academy of Sciences, Brno, Czech Republic. ${ }^{10}$ Bioscience Heart Failure, Cardiovascular, Renal \\ and Metabolism, Innovative Medicines and Early Development (IMED) Biotech Unit, AstraZeneca, Gothenburg, Sweden. \\ ${ }^{11}$ Department of Cellular and Molecular Medicine, Cleveland Clinic, Cleveland, Ohio, USA. ${ }^{12}$ Universities of Giessen and \\ Marburg Lung Center (UGMLC), Member of the German Center for Lung Research, Giessen, Germany.
}

Pulmonary arterial hypertension (PAH) remains a disease with limited therapeutic options and dismal prognosis. Despite its etiologic heterogeneity, the underlying unifying pathophysiology is characterized by increased vascular tone and adverse remodeling of the pulmonary circulation. Myeloperoxidase (MPO), an enzyme abundantly expressed in neutrophils, has potent vasoconstrictive and profibrotic properties, thus qualifying as a potential contributor to this disease. Here, we sought to investigate whether MPO is causally linked to the pathophysiology of PAH. Investigation of $\mathbf{2}$ independent clinical cohorts revealed that MPO plasma levels were elevated in subjects with PAH and predicted adverse outcome. Experimental analyses showed that, upon hypoxia, right ventricular pressure was less increased in $\mathrm{Mpo}^{-/-}$than in WT mice. The hypoxiainduced activation of the Rho-kinase pathway, a critical subcellular signaling pathway yielding vasoconstriction and structural vascular remodeling, was blunted in $\mathrm{Mpo}^{-/-}$mice. Mice subjected to i.v. infusion of MPO revealed activation of Rho-kinase and increased right ventricular pressure, which was prevented by coinfusion of the Rho-kinase inhibitor Y-27632. In the Sugen5416/hypoxia rat model, PAH was attenuated by the MPO inhibitor AZM198. The current data demonstrate a tight mechanistic link between MPO, the activation of Rho-kinase, and adverse pulmonary vascular function, thus pointing toward a potentially novel avenue of treatment.

Authorship note: AK, EB, and KF contributed equally to this work. VR and SB contributed equally to this work.

Conflict of interest: EM is employed by AstraZeneca.

Submitted: September 15, 2017

Accepted: April 19, 2018

Published: June 7, 2018

Reference information:

JCI Insight. 2018;3(11):e97530.

https://doi.org/10.1172/jci.

insight. 97530

\section{Introduction}

Pulmonary hypertension, defined as a mean pulmonary artery (PA) pressure of $>25 \mathrm{mmHg}$, can occur as a consequence of primary disease of the pulmonary vasculature, termed pulmonary arterial hypertension (PAH, Nice group 1) or a secondary manifestation of a variety of pulmonary or extrapulmonary disorders (Nice groups 2-5) (1). All forms of pulmonary hypertension involve (a) morphological changes, including vessel wall thickening and overt muscularization, and (b) functional alterations, such as impaired vasorelaxation and increased vasoconstriction (2). The relative impact of both components varies considerably between the different forms of pulmonary hypertension, with morphological changes being more pronounced in PAH (e.g., hyperproliferative, so called plexiform lesions, are only found in this group) and functional changes being of greater relevance (e.g., in hypoxia-related PAH). 
One of the principal unifying pathological processes underlying these disease mechanisms is disturbed pulmonary arterial endothelial and smooth muscle cell (SMC) signaling (3), a phenomenon ineffectively addressed with current pharmacological interventions. There is growing evidence that inflammation contributes to this process in PAH (4). Besides increased levels of circulating proinflammatory cytokines, perivascular invasion of inflammatory cells occurs in affected lung tissue from PAH patients (5).

The leukocyte-derived heme-enzyme myeloperoxidase (MPO) emerges as a potential mediator of PAH, as it is firmly established as a central mediator of humoral dysfunction of the vessel wall. MPO is abundantly expressed and released by polymorphonuclear neutrophils (PMN) and catalyzes the generation of bactericidal oxidative molecules like hypochlorous acid. However, it became obvious that MPO deficiency is not related to immune dysfunction (6); thus, MPO inhibition emerges as a potential therapeutic strategy. The enzyme has not only been shown to be a powerful predictor of clinical outcome in patients with cerebrovascular (7), peripheral vascular (8), and coronary vascular disease $(9,10)$, but it has also been identified to be intimately involved in the pathophysiology of vascular disease in general (11). These effects are in large part related to MPO's ability to reduce the bioavailability of nitric oxide (NO), which is an important antiinflammatory and vasodilating molecule (12). MPO binds with high affinity to the vessel wall due to its cationic charge and is transcytosed by endothelial cells to the subendothelial space (13). MPO is not only capable of oxidizing NO itself $(12,14)$, but it also decreases its bioavailability by oxidatively modifying NO-synthase (NOS) substrates and enzymes that are responsible for the degradation of endogenous NOS inhibitors $(15,16)$. A recent study including individuals with congenitally low MPO levels substantiated these findings by demonstrating a profound impact of MPO on vasomotor function (17).

Given increased activation of leukocytes and evidence for inflammation-dependent vascular dysfunction in PAH, we investigated the role of MPO in this disease.

\section{Results}

$M P O$ is increased in lung specimens, and circulating MPO levels predict outcomes in patients with $P A H$. In order to test whether PAH is associated with elevated secretion and pulmonary deposition of MPO, analyses of local deposition and systemic sequestration of MPO in patients with PAH were performed. Immunohistochemical imaging of lung specimens from PAH patients revealed enhanced immunoreactivity for MPO in lung sections as compared with controls (Figure 1A). In addition, 2 cohorts of a total of 95 patients with confirmed, prevalent $\mathrm{PAH}$ (mean age $50 \pm 14$ years, $78 \%$ female, Table 1) and 74 age and sex matched controls were investigated. Compared with controls, PAH patients exhibited significantly elevated MPO plasma levels (431 [interquartile range (IQR), 301-605] vs. 547 [IQR, 347-894] pmol/1, $P=0.006$, Figure 1B). A good correlation was observed for MPO and neutrophil elastase plasma levels, supporting that elevated MPO was a consequence of neutrophil degranulation ( $\rho=0.50, P<0.01$ ). PAH patients were followed for a median of 65 [IQR, 27-94] weeks. ROC curve analysis identified a cut-off of $583 \mathrm{pmol} / 1$ as the best cut-off for predicting survival. Using this cut-off level, PAH patients with high MPO plasma levels revealed decreased survival compared with those with low MPO levels (Figure $1 \mathrm{C}, \chi^{2}=5.134, P=0.023$ ). Although brain natriuretic peptide (BNP) plasma levels were also associated with decreased survival in PAH patients $\left(\chi^{2}=7.03, P<0.01\right)$, MPO predicted survival independently of BNP levels (MPO hazard ratio 3.1 [95\% CI, $1.1-8.7], P=0.035$ ). There was a trend toward a lower 6-minute-walk distance (6MWD) at baseline among patients with high MPO plasma levels (350 \pm 139 vs. $398 \pm 124$ $\mathrm{m}, P=0.104)$ and toward a negative correlation between MPO plasma levels and 6MWD distance $(\rho=-0.216$, $P=0.053$ ), indicating that MPO is associated with enhanced functional impairment in PAH.

$\mathrm{Mpo}^{--}$mice are protected from hypoxia-induced pulmonary hypertension. To test for a causal relation between MPO and PAH, we exposed C57BL/6J WT and MPO-deficient ( $\left.\mathrm{Mpo}^{--}\right)$mice to normobaric hypoxia $\left(10 \% \mathrm{O}_{2}\right)$. After 28 days, circulating plasma levels and pulmonary deposition of MPO were markedly increased in WT mice, which is a readout of activation and degranulation of PMN (Figure 2A and Supplemental Figure 1, A and B; supplemental material available online with this article; https://doi.org/10.1172/jci.insight.97530DS1). In order to assess whether liberated MPO modulates PA pressure, systolic right ventricular pressure (RVPsys) was determined after 28 days of hypoxia. In normoxic mice, there were no differences in RVPsys between WT and $\mathrm{Mpo}^{-/-}$mice. Hypoxia led to a significant increase of RVPsys in both WT and $\mathrm{Mpo}^{-/-}$mice. Of note, $\mathrm{Mpo}^{-/-}$mice exhibited a profoundly reduced RVPsys in comparison with WT mice (Figure 2B). These differences were corroborated by right-to-left ventricular mass ratios, which were similar between both groups for normoxia but were significantly higher for WT mice compared with $\mathrm{Mpo}^{-/-}$mice upon hypoxia (Figure 2C). 
A
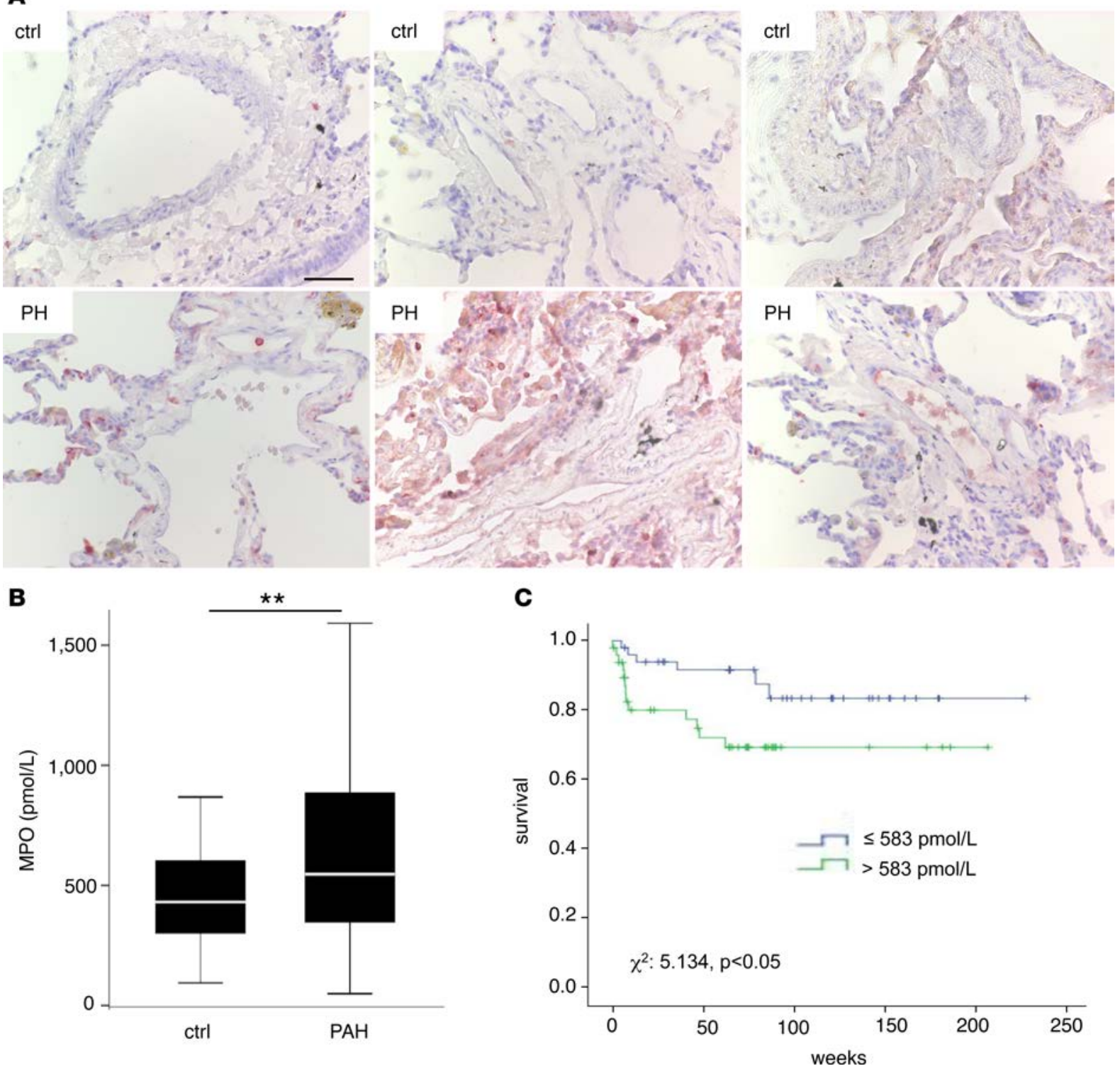

Figure 1. Myeloperoxidase in patients with pulmonary arterial hypertension. Myeloperoxidase (MPO) accumulates in plasma and lung tissue of patients with pulmonary arterial hypertension (PAH) and is related to increased mortality. (A) Immunohistochemical staining of MPO in lung sections of control subjects and patients with pulmonary hypertension (PH). Representative images of 3 separate individuals per group are displayed. Scale bar: $50 \mu \mathrm{m}$. (B) MPO plasma levels in PAH patients at baseline. $n=95 ;{ }^{*} P<0.01$. Data represent median, with interquartile range and whiskers indicating 5 th to 95 th percentile. Statistical analysis was performed by Mann-Whitney $U$ test. (C) Kaplan-Meier analysis of survival of PAH patients with MPO plasma levels $<583$ $\mathrm{pmol} / \mathrm{l}$ (low) or $>583 \mathrm{pmol} / \mathrm{l}$ (high). $n=95 ; \chi^{2}=5.134, P<0.05$. Statistical analysis was performed by log-rank test.

To further characterize the hypoxia-induced inflammatory response, MPO levels were also assessed in the early phase of hypoxia. After 2 hours of hypoxia, pulmonary MPO deposition, but not plasma MPO levels, were increased in WT mice as compared with normoxic WT animals, and no differences were detected in circulating WBC counts (Supplemental Figure 1, A-C). Moreover, pulmonary PMN infiltration was higher than in normoxic animals in both WT and $\mathrm{Mpo}^{-/-}$mice after 2 hours and was significantly lower in $\mathrm{Mpo}^{-/-}$than in WT animals after 28 days of hypoxia (Supplemental Figure 1D). Apart from that, mRNA of inflammation-related genes in lung tissue was quantified after 2 hours of hypoxia, and pro- and antiinflammatory cytokines were analyzed in lung homogenates from mice after 2 and 4 hours and 28 days of hypoxia. No significant differences were detectable between WT and $\mathrm{Mpo}^{-/-}$mice after normoxia or in the early or chronic phases of hypoxia (except from a significantly higher level of granulocyte-macrophage $\mathrm{CSF}$ in WT as compared with $\mathrm{Mpo}^{-/-}$mice upon 2 hours of hypoxia) (Supplemental Figures 2 and 3). 
Table 1. Patient characteristics

\begin{tabular}{|c|c|c|c|}
\hline \multicolumn{4}{|c|}{ Clinical baseline characteristics ( $n=95$ ) } \\
\hline Age, yrs & $48.9 \pm 15.4$ & $51.0 \pm 13.3$ & 0.47 \\
\hline Female & $37(77)$ & $41(87)$ & 0.20 \\
\hline IPAH & $33(69)$ & $19(40)$ & 0.03 \\
\hline FPAH & $5(10)$ & $3(6)$ & \\
\hline Other & $1(2)$ & $2(4)$ & \\
\hline Endothelin 1 antagonist & $35(73)$ & $28(60)$ & 0.17 \\
\hline PDE5 inhibitor & $25(46)$ & $29(54)$ & 0.34 \\
\hline Prostacyclin analogue & $36(75)$ & $28(57)$ & 0.12 \\
\hline \multicolumn{4}{|l|}{ Combination therapy } \\
\hline Cardiac index, $1 / \mathrm{min} / \mathrm{m}^{2}$ & $2.4 \pm 0.9$ & $2.4 \pm 1.0$ & 0.81 \\
\hline PVRI, dyn $\times s / \mathrm{cm}^{5} \times \mathrm{m}^{2}$ & $1,692 \pm 948$ & $1,650 \pm 838$ & 0.83 \\
\hline $\mathrm{PAC}, \mathrm{ml} / \mathrm{mmHg}$ & $1.31 \pm 0.70$ & $1.30 \pm 1.07$ & 0.96 \\
\hline WBC count, $\times 10^{6} / \mathrm{ml}$ & $7.7 \pm 3.7$ & $7.7 \pm 3.8$ & 0.99 \\
\hline BNP & $1,084 \pm 2,517$ & $2,076 \pm 7,889$ & 0.42 \\
\hline CRP, mg/dl & $3.1 \pm 6.1$ & $2.1 \pm 2.8$ & 0.65 \\
\hline
\end{tabular}

PAH, pulmonary arterial hypertension; IPAH, idiopathic PAH; FPAH, familial PAH; PA, pulmonary artery; PC, pulmonary capillary wedge; PVRI, pulmonary vascular resistance index; PAC, pulmonary arterial compliance; WBC, white blood count; BNP, B-natriuretic peptide; CRP, C-reactive protein.

MPO has no effect on NO-dependent pulmonary vasorelaxation. Given its known effects on decreasing NO bioavailability in various vascular beds, we first investigated the effects of MPO on pulmonary arterial NO bioavailability. Unexpectedly, acetylcholine-induced (ACh-induced) vasorelaxation was unaffected by MPO after 28 days of hypoxia (Figure 3A), suggesting similar local NO bioavailability between the different groups. Moreover, the amount of phosphorylated vasodilator-stimulated phosphoprotein (p-VASP) related to total VASP - a readout of NO bioavailability - was quantified in lung homogenates. Whereas phosphorylation of VASP tended to be increased in $\mathrm{Mpo}^{-1-}$ as compared with WT mice under normoxia, similar levels of p-VASP in both groups following 28 days of hypoxia were observed (Figure 3B). Interestingly, protein levels of inducible NOS (iNOS) in lung homogenates were increased following hypoxia in WT but not in $\mathrm{Mpo}^{--}$mice (Figure 3C).

MPO increases pulmonary vasoconstriction in vivo and ex vivo via Rho-kinase. To test whether MPO exerts a vasoconstrictive effect on the pulmonary vasculature, we exposed isolated pulmonary artery (PA) segments to prostaglandin $\mathrm{F}_{2} \alpha\left(\mathrm{PGF}_{2} \alpha\right)$. Segments of both WT and $\mathrm{Mpo}^{-/-}$mice exhibited a profoundly increased vasoconstrictive response after merely 7 days of hypoxia compared with normoxic animals. However, this response was significantly more pronounced in WT than $\mathrm{Mpo}^{-/-}$mice (Figure 4A). To further corroborate the role of MPO-dependent vasoconstriction in PAH, the effective arterial elastance ( $\mathrm{E}_{\mathrm{a}}$ )was assessed from right ventricular pressure-volume loops. The effective $\mathrm{E}_{\mathrm{a}}$ represents an established parameter of ventricular afterload, thus reflecting vascular resistance and compliance (18). Of note, $\mathrm{E}_{\mathrm{a}}$ was significantly increased in WT mice upon 28 days of hypoxia (19), but was profoundly diminished in $\mathrm{Mpo}^{-/-}$mice (Figure 4B).

Immunohistochemical studies revealed increased vascular muscularization of pulmonary arterioles of both WT and $\mathrm{Mpo}^{-/-}$mice held under hypoxic conditions for 28 days as compared with mice under normoxic conditions. However, no significant differences were detectable between WT and $\mathrm{Mpo}^{-/-}$mice upon hypoxia (Figure 4, C and D). Since activation of the ras homolog family member A (RhoA)/Rho-kinase pathway is not only a central mediator of vasoconstriction in PAH but has also been shown to be under redox-sensitive control (20), we investigated its role in MPO-dependent pulmonary vasoconstriction. Mice 
A
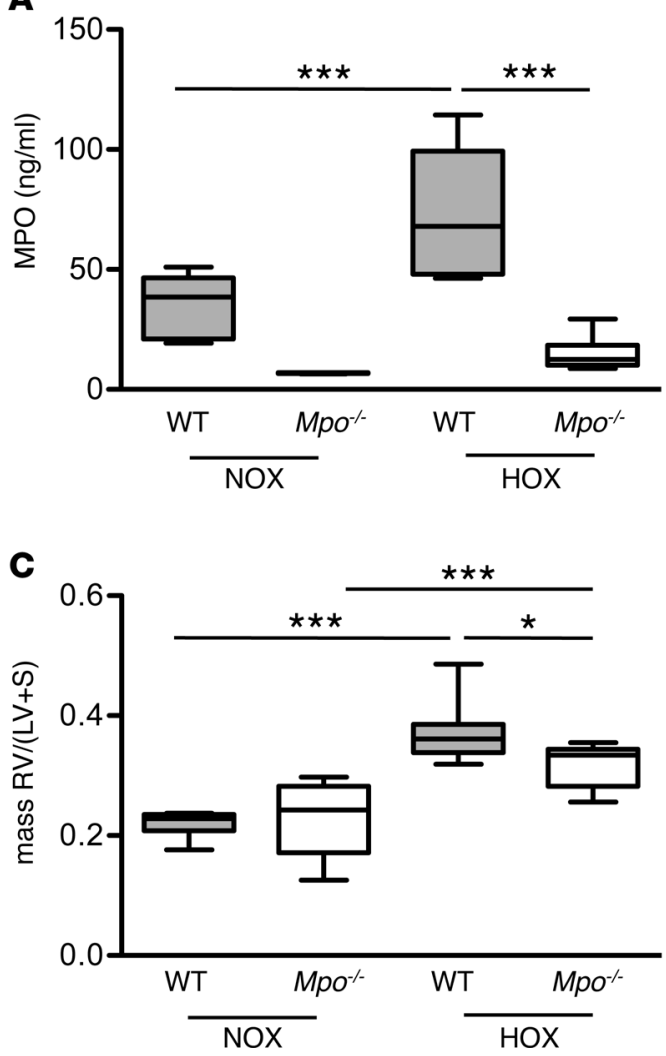

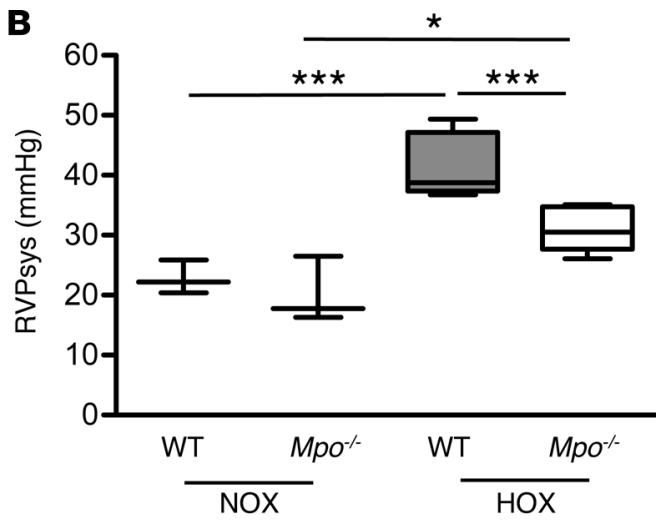

Figure 2. Impact of myeloperoxidase on hypoxia-induced pulmonary hypertension in mice. Hypoxia leads to increased pulmonary hypertension in WT but not $\mathrm{MpO}^{-/-}$mice. Animals were maintained for 28 days under normoxic (NOX) or hypoxic $\left(10 \% \mathrm{O}_{2}, \mathrm{HOX}\right)$ conditions. (A) MPO plasma levels of WT and $\mathrm{Mpo}^{-/-}$mice after 28 days of NOX or HOX, as determined by ELISA. $n=6$ (WT NOX), 4 (Mpo ${ }^{-1-}$ NOX), 8 (WT HOX), 6 (Mpo ${ }^{-1-} \mathrm{HOX}$ ); ***P<0.001. (B) Right ventricular systolic pressure (RVPsys) in WT and $\mathrm{Mpo}^{-/-}$mice upon 28 days of NOX or HOX was assessed using a microtip catheter. $n=3$ (WT, Mpo ${ }^{-/-}$NOX), 8 (WT, Mpo ${ }^{-1-} \mathrm{HOX}$ ); ${ }^{*} P<0.05,{ }^{* * *} P<0.001$. (C) Right ventricular hypertrophy as expressed by mass ratio of right ventricle to left ventricle and septum $(\mathrm{RV} /[\mathrm{LV}+\mathrm{S}])$ in WT and $\mathrm{Mpo}^{-/-}$mice. $n=8$ (WT NOX, HOX), $6\left(\mathrm{Mpo}^{-/-} \mathrm{NOX}\right), 8\left(\mathrm{Mpo}^{-1-} \mathrm{HOX}\right) ;{ }^{*} P<0.05,{ }^{* *} P<0.001$. Data represent median with interquartile range; whiskers indicate minimum to maximum. Statistical analysis was performed with 1-way ANOVA followed by LSD post hoc test.

were treated with the Rho-kinase inhibitor Y-27632 one hour prior to isolation of the PA. Constriction of PA segments of hypoxic WT mice was significantly attenuated upon treatment with Y-27632 and was reduced to the level observed in untreated hypoxic $\mathrm{Mpo}^{-/-}$mice, whereas Y-27632 treatment exerted no additive effect in hypoxic $\mathrm{Mpo}^{--}$animals (Figure 4A).

MPO activates the RhoA/Rho-kinase pathway in vivo and in vitro. Following the above described functional analyses, Rho-kinase activity was determined in lung tissue homogenates by immunoblot analysis of phosphorylated myosin phosphatase target subunit-1 (p-MYPT-1). Indeed, lung homogenates of WT mice displayed markedly elevated levels of p-MYPT-1 upon 28 days of hypoxia as compared with normoxic mice, whereas Rho-kinase activity was significantly lower in lung homogenates of hypoxic $\mathrm{Mpo}^{-/}$mice (Figure 5A). In contrast, no significant differences were observed in the Rho-kinase mRNA expression in lung tissue of WT and $\mathrm{Mpo}^{-1-}$ mice (Figure 5B).

To further confirm MPO-dependent regulation of Rho-kinase activity, in vitro experiments using human PA smooth muscle cells (PASMCs) were performed. Exposure of cells to enzymatically active MPO increased phosphorylation of MYPT-1, whereas the catalytically inactive MPO variant Q91T had no effect (Figure 5C). Given that the small G-protein RhoA, which is an upstream stimulator of Rho-kinase, is activated by ROS (20), its activity was analyzed in PASMCs using a small G-protein activation assay. Indeed, treatment of PASMCs with active MPO but not with inactive Q91T MPO resulted in a dose-dependent activation of RhoA (Figure 5D).

I.v. application of MPO elicits pulmonary hypertension. To further corroborate the effects of MPO on Rho-kinase signaling in vivo, WT and $\mathrm{Mpo}^{-/-}$mice were i.v. infused with human recombinant MPO for 7 days (21). MPO infusion resulted in significantly elevated RVPsys in mice, which was normalized upon 

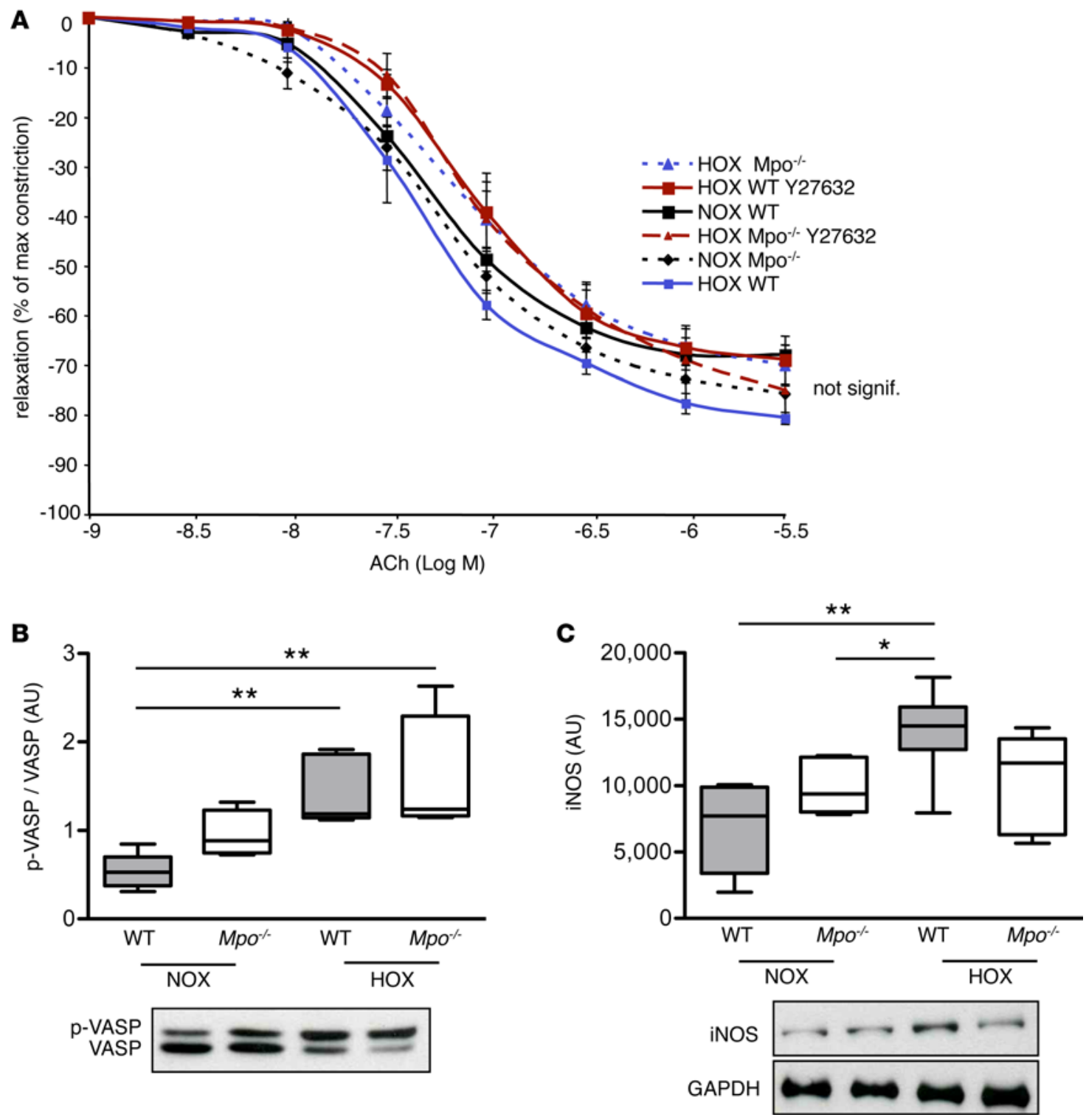

Figure 3. Pulmonary nitric oxide bioavailability. Pulmonary artery nitric oxide (NO) bioavailability is not affected in $\mathrm{Mpo}^{-/-}$mice upon hypoxia (HOX). (A) Mice were maintained for 28 days under normoxia (NOX) or $\operatorname{HOX}\left(10 \% \mathrm{O}_{2}\right)$, and the Rho-kinase inhibitor Y-27632 was administered 1 hour prior to sacrifice. Relaxation of explanted pulmonary arteries in response to acetylcholine (ACh) was assessed by isometric force measurements and expressed as \% of

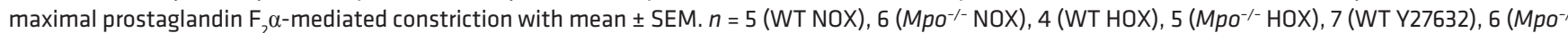
Y27632). Statistical analysis was performed by 1-way ANOVA for repeated measures. Differences are not statistically significant. (B) Lung homogenates of mice after 28 days of NOX or HOX were analyzed by Western blot, and protein amount of phosphorylated vasodilator stimulated phosphoprotein ( $p$-VASP, Ser-157) in relation to dephosphorylated VASP was assessed. $n=5$ (WT NOX), 4 (Mpo-/- NOX), 5 (WT HOX), 4 (Mpo ${ }^{-/-}$HOX); **P $<0.01$. (C) Protein amount of inducible NO-synthase (iNOS) related to GAPDH was assessed in lung homogenates of mice after 28 days of NOX or HOX. $n=5$ (WT NOX), 5 (Mpo $0^{-/-}$NOX), 6 (WT HOX), $5\left(\mathrm{Mpo}^{-/-} \mathrm{HOX}\right) ;{ }^{*} P<0.05,{ }^{* *} P<0.01$. Data in $\mathbf{B}$ and $\mathbf{C}$ represent median with interquartile range; whiskers indicate minimum to maximum. Statistical analysis was performed with 1-way ANOVA with LSD post hoc test.

coinfusion with the Rho-kinase inhibitor Y-27632 (Figure 6A). Accordingly, MPO infusion led to increased Rho-kinase activity in lung homogenates of WT and $\mathrm{Mpo}^{-/-}$mice, which was blunted by coinfusion of Y-27632 (Figure 6B). Unlike the observations made under hypoxic conditions, MPO provoked a significant increase in muscularization of vessels with small diameters $(20-70 \mu \mathrm{m})$ (Figure 6, C and D). Of note, MPO infusion induced a profound pulmonary infiltration of PMN (Supplemental Figure 4). Activated PMN can serve as the source of hydrogen peroxide, which is required for catalytic activity of infused MPO.

Administration of the MPO inhibitor AZM198 attenuates pulmonary hypertension and pulmonary vascular remodeling in SuHx-treated rats. In order to examine the effects of MPO inhibition in a model of PAH which even better reflects human disease, particularly with regard to vascular remodeling $(22,23)$ - we 


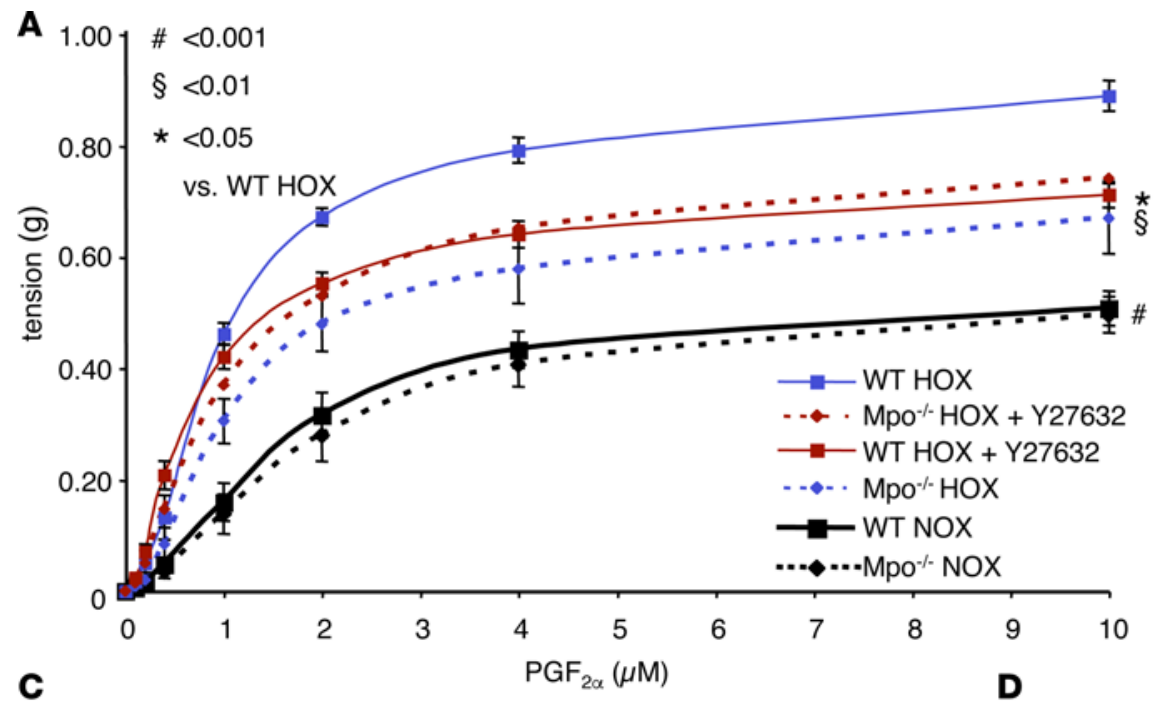

B
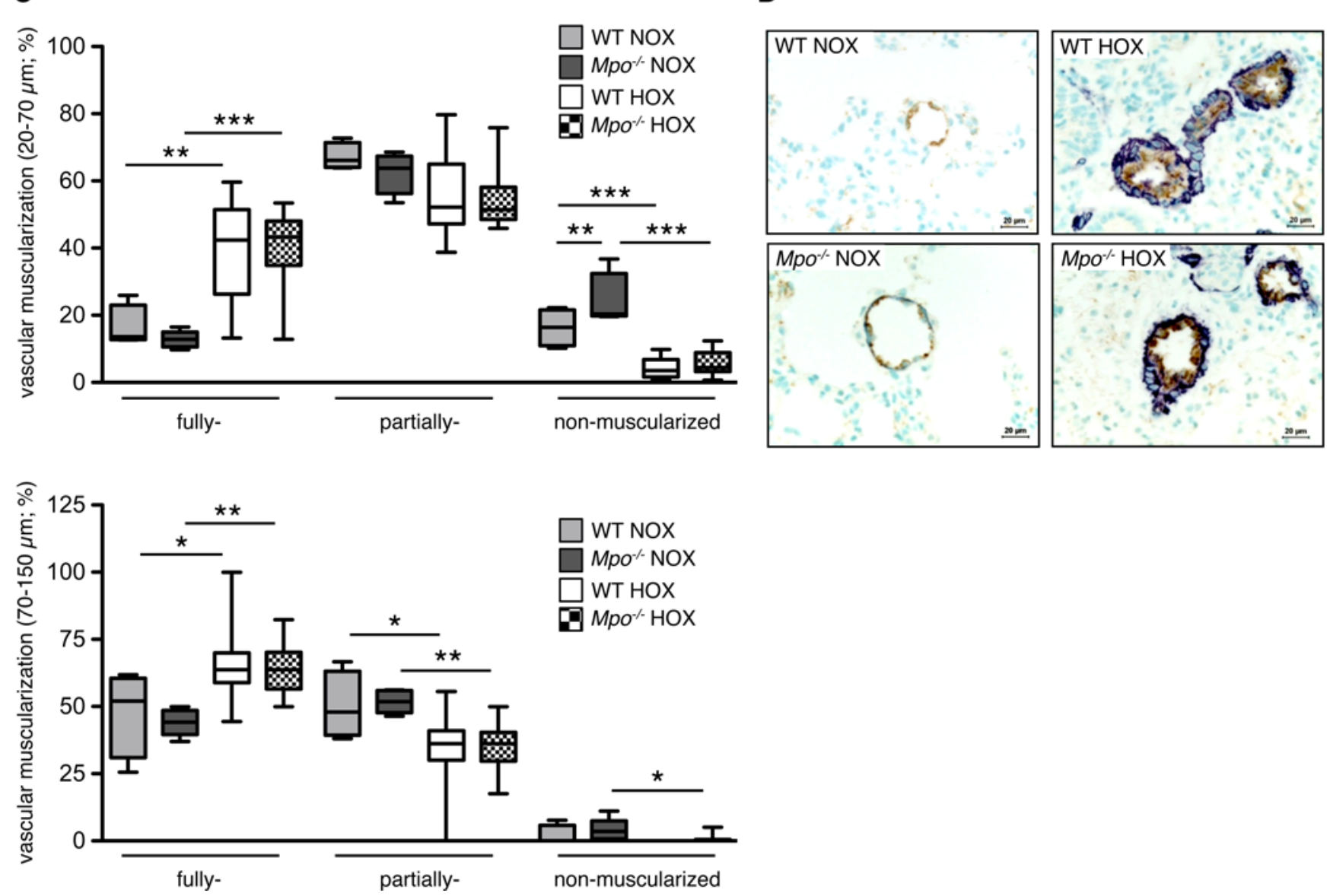

Figure 4. Pulmonary vascular constriction. Hypoxia-induced pulmonary vascular constriction and arterial elastance is less pronounced in $\mathrm{Mpo}^{-/-}$as compared with WT mice, whereas vascular muscularization is not affected by myeloperoxidase (MPO). (A) Mice were kept under normoxia (NOX) or hypoxia $\left(10 \% \mathrm{O}_{2}, \mathrm{HOX}\right)$ for 7 days, and the Rho-kinase inhibitor $\mathrm{Y} 27632$ was administered i.p. 1 hour prior to sacrifice. Constriction of isolated pulmonary artery segments in response to increasing concentrations of prostaglandin $\mathrm{F}_{2} \alpha\left(\mathrm{PGF}_{2} \alpha\right)$ was assessed by isometric force measurements. $n=5$ (WT NOX),

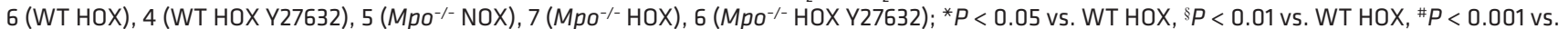
WT HOX. Data represent mean \pm SEM. (B) Effective arterial elastance $\left(E_{a}\right)$ of WT and $M p 0^{-/-}$mice after 28 days of NOX or HOX was calculated from right ventricular pressure-volume analyses. $n=3$ (WT, $\mathrm{Mpo}^{-/-} \mathrm{NOX}$ ), 8 (WT HOX), 9 ( $\mathrm{Mpo}^{-1-} \mathrm{HOX}$ ); ${ }^{*} P<0.05,{ }^{* *} P<0.01$. (C) Percentages of nonmuscularized, partially muscularized, and fully muscularized pulmonary arteries of diameters of 20-70 $\mu \mathrm{m}$ and $70-150 \mu \mathrm{m}$ were assessed in immunohistochemically stained lung sections from mice after 28 days of NOX and HOX. $n=4$ (WT NOX), 5 (Mpo ${ }^{-/-}$NOX), 12 (WT HOX), 14 (Mpo $\left.{ }^{-/-} \mathrm{HOX}\right) ;{ }^{*} P<0.05,{ }^{* *} P<0.01$, ${ }^{* *} P<0.001$. (D) Representative images of lung sections: brown, von Willebrand factor; dark blue, $\alpha$-smooth muscle actin. Scale bar: $20 \mu \mathrm{m}$. Data in $\mathbf{B}$ and $\mathbf{C}$ represent median with interquartile range; whiskers indicate minimum to maximum. Statistical analysis was performed for $\mathbf{A}$ with 1 -way ANOVA for repeated measures and, for $\mathbf{B}$ and $\mathbf{C}$, with 1-way ANOVA followed by LSD post hoc test. 
utilized the model of Sugen5416/hypoxia-induced (SuHx-induced) pulmonary hypertension in rats and fed them with an MPO inhibitor-containing chow (AZM198) or control chow (Figure 7A).

As expected, SuHx caused a marked increase of vascular muscularization in small PAs of control chow-treated animals. Treatment with AZM198 diminished this vascular remodeling, as the percentage of fully muscularized vessels was significantly reduced in the treatment group (Figure 7, B and C). Consistently, RVPsys and RV hypertrophy were increased in the control chow-treated animals, which was significantly attenuated by AZM198 treatment (Figure 7, D and E). Of note, no differences were detected in systemic blood pressure (SBP) and heart rate (Figure 7, F and G). Thus, the effect of pharmacological inhibition of $\mathrm{MPO}$ in this rat model confirms the findings derived from the hypoxia model using $\mathrm{Mpo}^{-/-}$mice.

\section{Discussion}

The current data provide evidence for the leukocyte-derived enzyme MPO to be mechanistically linked to PAH: (a) lung specimens of PAH patients revealed increased MPO immunoreactivity, and circulating plasma levels of the enzyme were elevated in humans with $\mathrm{PAH}$ and predict clinical outcome; (b) mice deficient in MPO were protected from hypoxia-induced PAH and exaggerated vasoconstrictor response; (c) i.v. infusion of MPO, in the absence of further stimuli, is sufficient to induce PAH and to provoke pulmonary vascular remodeling; and (d) pharmacological MPO inhibition attenuated vascular muscularization and $\mathrm{PAH}$ in SuHx-treated rats. The in vivo experiments and analyses of harvested tissue reveal a central mechanistic role of Rho-kinase for MPO's effects on the pulmonary vasculature, which is corroborated by in vitro studies demonstrating direct activation of the Rho-kinase pathway by MPO.

Whereas the contributory role of MPO in vascular disease so far has been mainly related to its lipid-oxidizing (24), cytokine-like (25), and NO-scavening effects $(12,17)$, this study demonstrates that MPO promotes functional and structural vascular remodeling in the pulmonary circulation via a mechanism so far not linked to MPO's catalytic activity. The current findings identify activation of RhoA and subsequently Rho-kinase in response to MPO as an important mechanism yielding increased PA pressure, whereas pulmonary NO bioavailability was not critically altered by MPO. Interestingly, iNOS protein expression in lung tissue under hypoxia was markedly increased in WT mice but not altered in $\mathrm{Mpo}^{-/-}$mice, suggesting that enhanced iNOS-dependent NO generation counterbalanced by MPO-mediated NO oxidation (12) or NOS inhibition (16) resulted in similar levels of vascular NO in both animal groups. These findings are in line with previous observations, reporting upregulated iNOS in animal models of PAH upon chronic hypoxia $(26,27)$ and revealing that inflammatory mediators and ROS relate to increased iNOS expression $(28,29)$. Furthermore, attenuated pulmonary PMN infiltration in $\mathrm{Mpo}^{-/-}$mice may contribute to the difference in iNOS levels.

The clinical benefits observed in allocating local NO bioavailability are viewed as an expression of augmented NO demand due to the increased release of vasoconstrictors like thromboxane, endothelin-1, or serotonin in PAH (30). In this regard, Rho-kinase is considered a central hub for these mediators, which contribute to functional impairment of the vasculature in PAH (31). Rho-kinase activation mediates SMC proliferation; the precise mechanisms underlying this process remain unclarified $(32,33)$. Even more important, Rho-kinase leads to inhibition of myosin light chain phosphatase with subsequent elevation of myosin light chain phosphorylation. This results in an increased responsiveness of smooth muscle myofilaments to $\mathrm{Ca}^{2+}(31)$. Thus, Rho-kinase activation provides a convincing mechanism for MPO-dependent vasoconstriction and vascular muscularization. Apart from receptor-mediated activation by the above-mentioned pathways, Rho-kinase indeed can be activated by virtue of its redox sensitivity (20). The PASMCs in vitro experiments using enzymatically active, as well as inactive, MPO clearly identify RhoA to be activated by MPO-derived reactive species with subsequent activation of Rho-kinase (Figure 8). These findings suggest that MPO may be a contributor of hypoxic pulmonary vasoconstriction (HPV), given the fact that, apart from the classical effect of hypoxia on ion channels, Rho-kinase activation has been suggested to be part of the HPV mechanism (34).

Limitations of the study. The main limitation of our study is that a model of hypoxia-induced pulmonary hypertension was used for characterization of our $\mathrm{KO}$ animals. Although commonly used, it is controversially discussed whether this model reflects PAH, particularly as morphological changes typically observed in humans with PAH are only marginal. In line with these considerations, we failed to show an effect of MPO on pulmonary vascular muscularization in this model. We therefore also employed a SuHx rat model, which is probably one of the best current animal models of PAH, as well as i.v. MPO infusion to elicit pulmonary hypertension. Indeed, both models were not only able to replicate the effects of MPO on pulmonary pressure, but also provide evidence for an MPO-dependent increase of pulmonary vascular muscularization. 

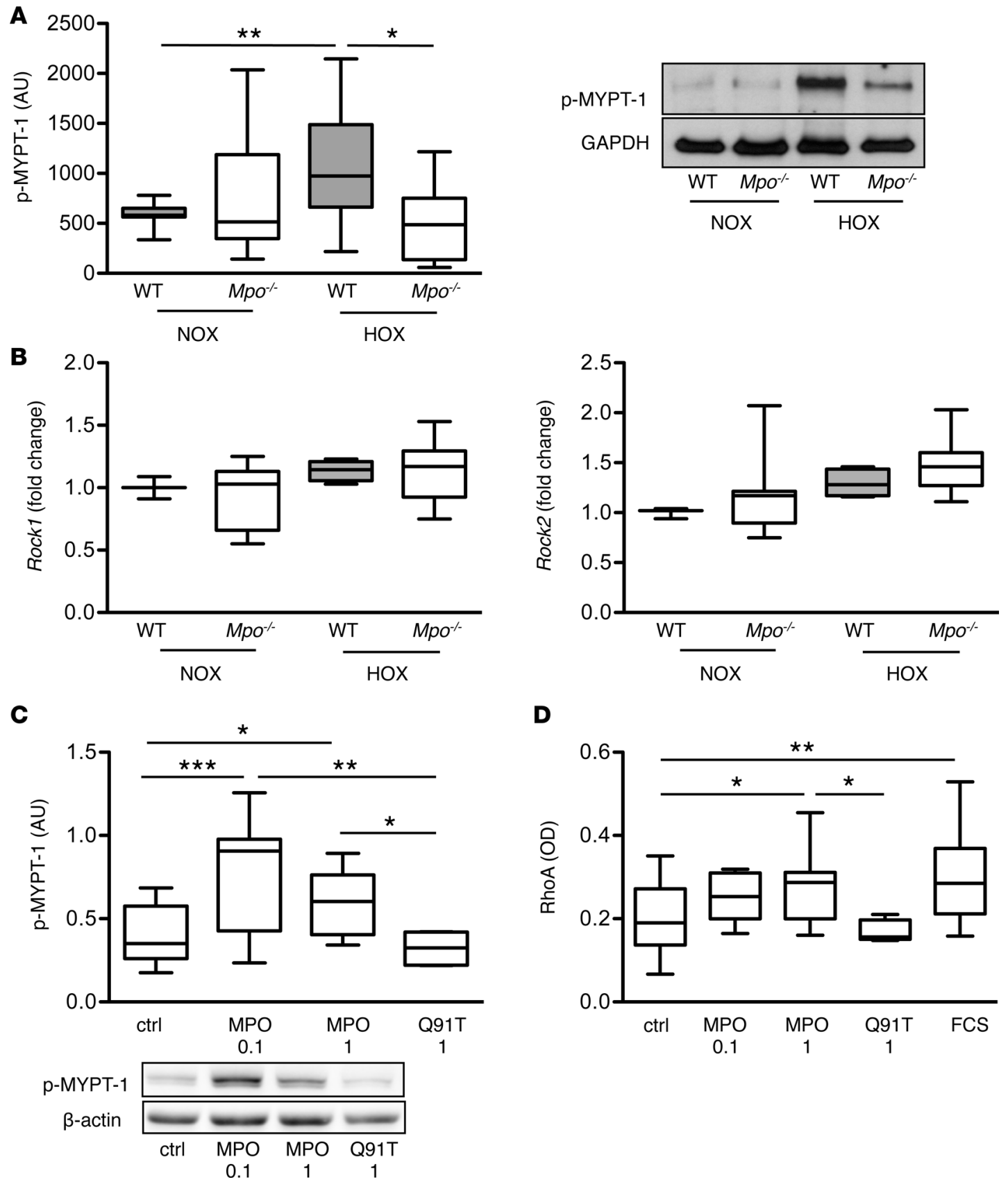

Figure 5. Rho-kinase and RhoA activation. Myeloperoxidase (MPO) mediates activation of Rho-kinase and RhoA in vivo and in vitro. (A) Western blots for phosphorylated myosin phosphatase target subunit-1 (p-MYPT-1) related to GAPDH reflecting Rho-kinase activity in lung homogenates of WT and $\mathrm{Mpo}^{-1-}$ mice upon 28 days of normoxia (NOX) or hypoxia $\left(10 \% \mathrm{O}_{2}, \mathrm{HOX}\right.$ ) were analyzed densitometrically. $n=16$ (WT NOX), 11 (Mpo-/- NOX), 15 (WT HOX), 6 ( $\left.\mathrm{Mpo}^{-1-} \mathrm{HOX}\right) ;{ }^{*} \mathrm{P}<0.05$, ${ }^{* *} \mathrm{P}<0.01$. (B) mRNA level of Rock1 and Rock2 in lung tissue of WT and Mpo-1- mice upon 7 days of NOX or HOX were assessed by quantitative PCR. $n=3$ (WT NOX), 4 (Mpo-/- NOX), 13 (WT HOX), 13 (Mpo (WOX). $^{-/ C}$ ) Pulmonary artery smooth muscle cells (PASMCs) were cultured and treated for 20 minutes with 0.1 or $1 \mu \mathrm{g} / \mathrm{ml} \mathrm{MPO}$ with hydrogen peroxide or with $1 \mu \mathrm{g} / \mathrm{ml}$ inactive MPO variant Q91T. Phosphorylated myosin phosphatase target subunit-1 (p-MYPT-1) related to $\beta$-actin reflecting Rho-kinase activity was quantified densitometrically. $n=14$ (ctrl), 8 (MPO 0.1), 12 (MPO 1), 4 (Q91T); 3 independent experiments; ${ }^{*} P<0.05,{ }^{*} P<0.01,{ }^{* * *} P<0.001$. (D) Cultured PASMCs were treated with 0.1 or $1 \mu \mathrm{g} / \mathrm{ml} \mathrm{MPO} \mathrm{with} \mathrm{hydrogen} \mathrm{peroxide,} 1 \mu \mathrm{g} / \mathrm{ml}$ inactive MPO variant Q91T, or FCS for $30 \mathrm{sec}$, harvested, and analyzed for active RhoA using a small GTPase activation assay. RhoA activity is expressed by OD. $n=30$ (ctrl), 5 (MPO 0.1), 8 (MPO 1), 5 (Q91T), 17 (FCS); 10 independent experiments; * $P<$ $0.05,{ }^{*} P<0.01$. Data are presented as median with interquartile range; whiskers indicate minimum to maximum. Statistical analysis was performed with 1-way ANOVA followed by LSD post hoc test. 
A

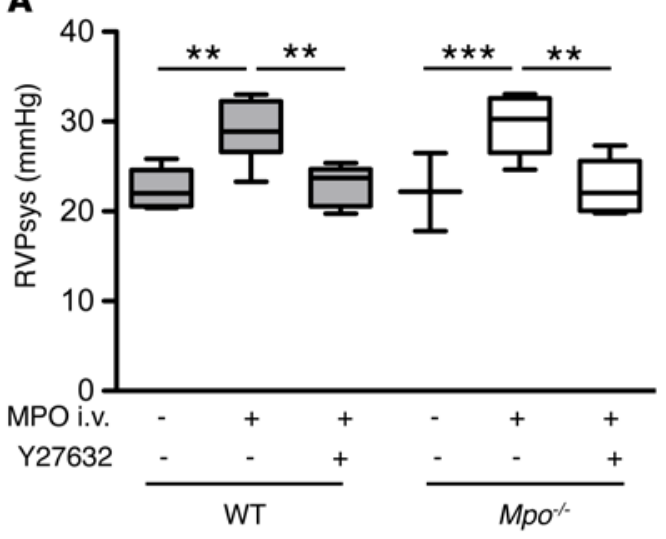

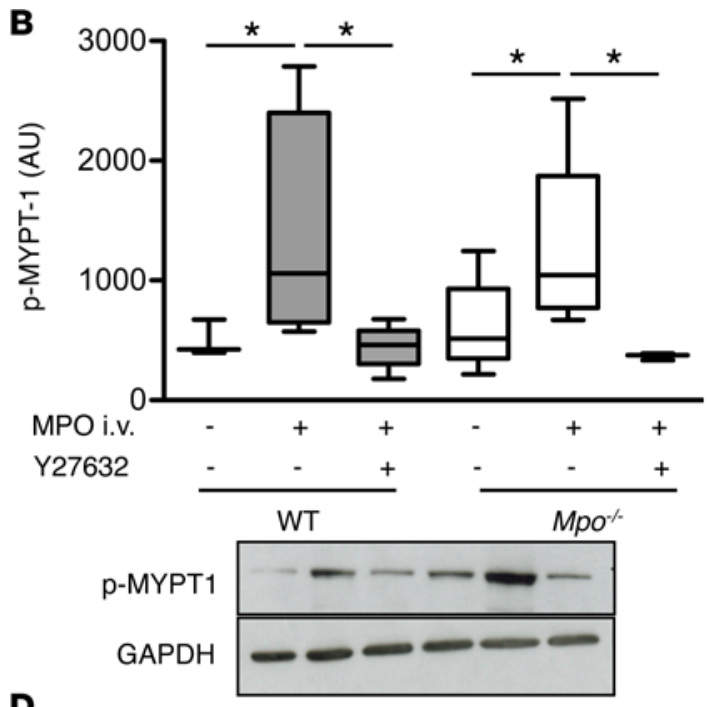

C

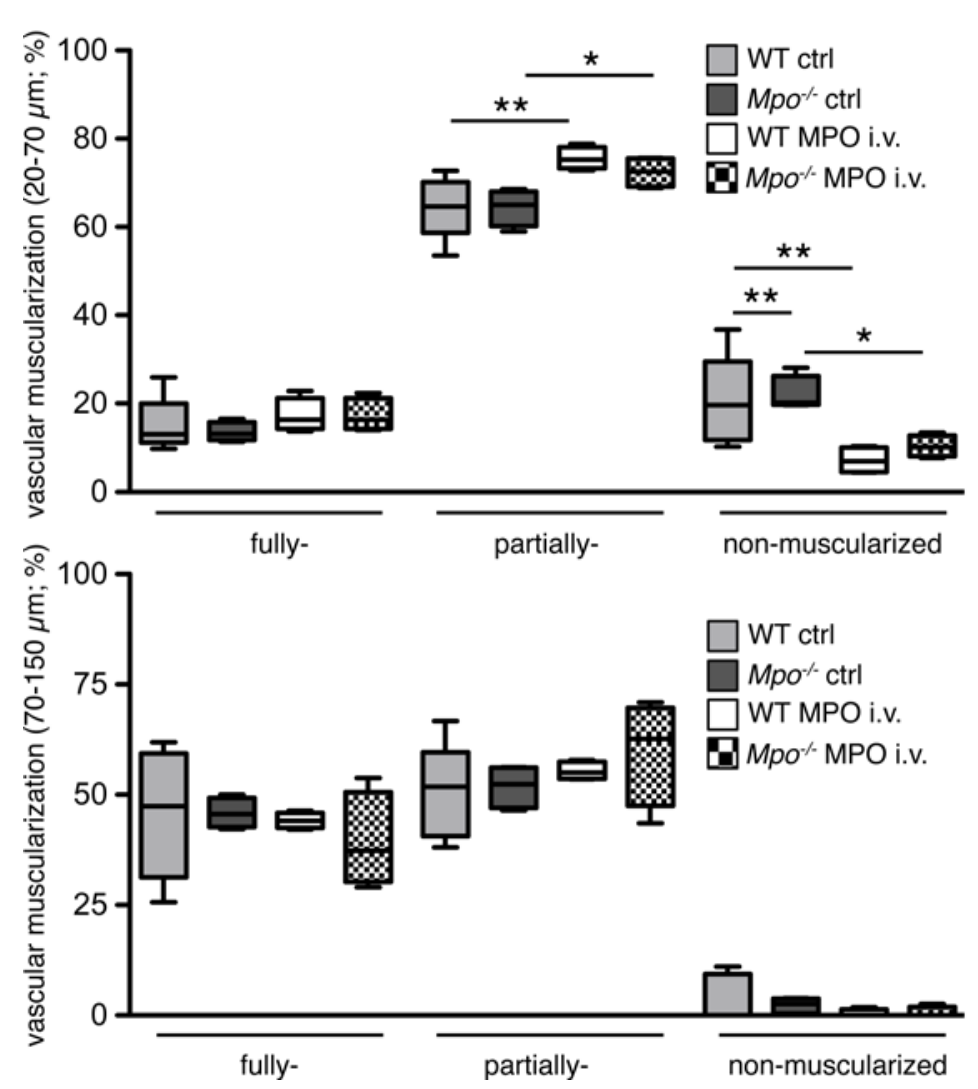

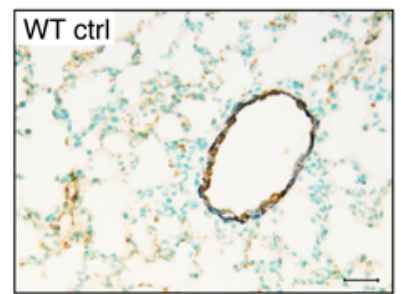
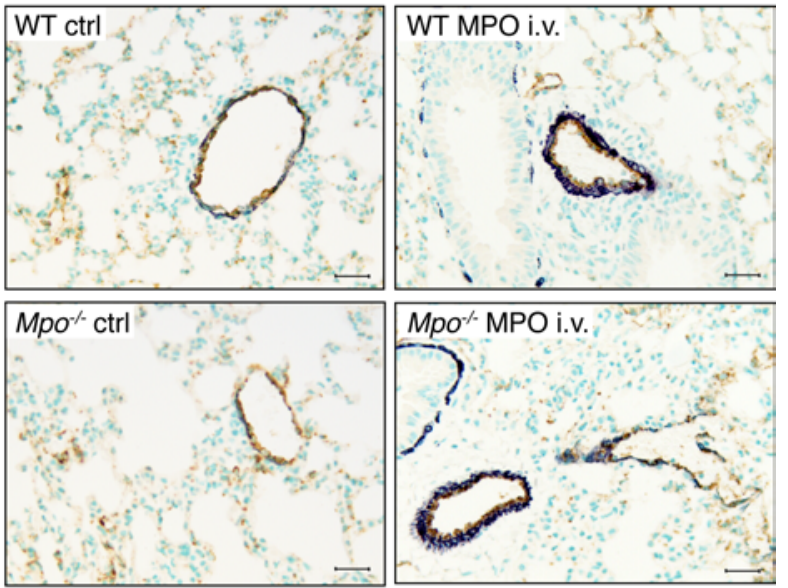

Figure 6. Pulmonary arterial hypertension upon infusion of myeloperoxidase. Myeloperoxidase (MPO) induces increase in right ventricular pressure and pulmonary artery muscularization in WT and $\mathrm{Mpo}^{-/-}$mice. (A) MPO or human serum albumin was infused to the jugular vein of WT and $\mathrm{Mpo}^{-/-}$ mice for 7 days using osmotic minipumps connected to a jugular vein catheter, and the Rho-kinase inhibitor Y27632 was infused s.c. for 7 days. Right ventricular systolic pressure (RVPsys) was assessed using a microtip catheter. $n=5$ (WT ctrl), 6 (WT MPO i.v.), 5 (WT MPO i.v. Y27632), 3 (Mpo-/- ctrl), 5 ( $\mathrm{Mpo}^{-/-} \mathrm{MPO}$ i.v.), $5\left(\mathrm{Mpo}^{-1-} \mathrm{MPO}\right.$ i.v. Y27632); ${ }^{* *} \mathrm{P}<0.01$, ${ }^{* *}{ }^{*} \mathrm{P}<0.001$. (B) Lung homogenates of mice after 7 days of MPO infusion with and without infusion of the Rho-kinase inhibitor Y27632 were analyzed for Rho-kinase activity by densitometrical quantification of Western blots for phosphorylated myosin phosphatase target subunit-1 (p-MYPT-1) related to GAPDH. $n=3$ (WT ctrl), 4 (WT MPO i.v.), 5 (WT MPO i.v. Y27632), 6 (Mpo-/- ctrl), 5 ( $\mathrm{Mpo}^{-/-} \mathrm{MPO}$ i.v.), $3\left(\mathrm{Mpo}^{-/-} \mathrm{MPO}\right.$ i.v. Y27632); ${ }^{*} P<0.05$. (C) Percentage of nonmuscularized, partially muscularized, and fully muscularized pulmonary arteries of diameters of 20-70 $\mu \mathrm{m}$ or 70-150 $\mu \mathrm{m}$ of WT and $\mathrm{Mpo}^{-/-}$mice was assessed in immunohistochemically stained lung sections from control mice or upon 7 days of i.v. MPO infusion. $n=5$ (WT ctrl), 4 (WT MPO i.v.), 4 (Mpo ${ }^{-1-}$ ctrl, MPO i.v.); ${ }^{*} P<0.05$, ${ }^{* *} P<0.01$. (D) Representative images of lung sections: brown, von Willebrand factor; dark blue, $\alpha$-smooth muscle actin. Scale bar: $20 \mu \mathrm{m}$. Data in A-C are presented as median with interquartile range; whiskers indicate minimum to maximum. Statistical analysis was performed with 1-way ANOVA followed by LSD post hoc test. 
A

Day 0

Day 21

Day 35

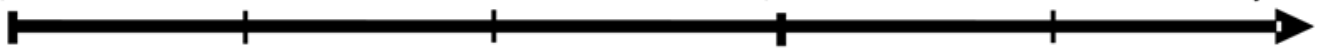

SU5416 (20 mg/kg)

AZM198 or control chow

Hypoxia

B

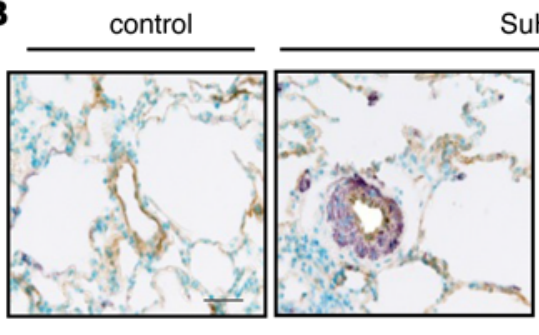

control chow
SuHx

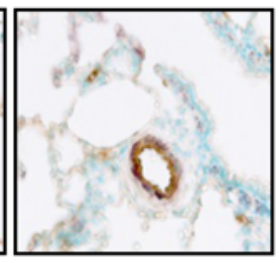

AZM198
D

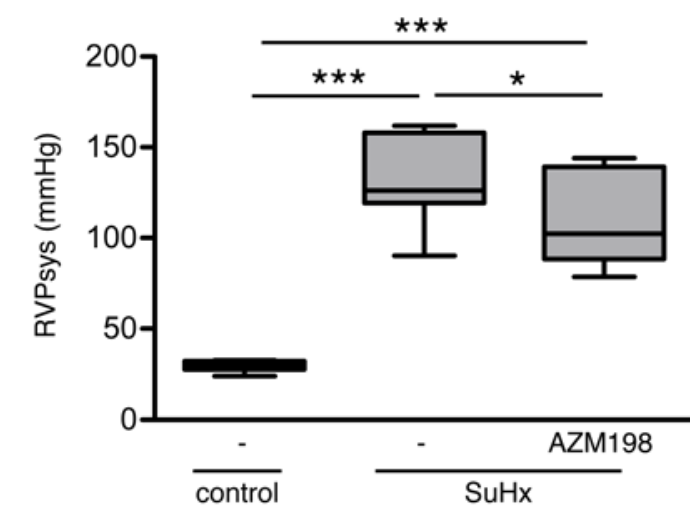

F

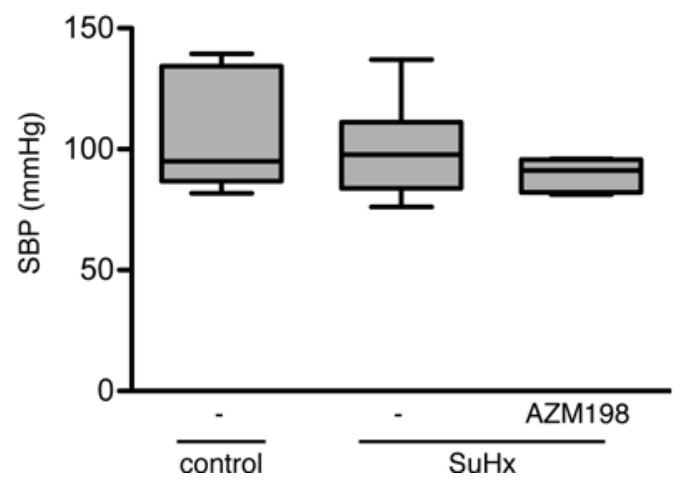

Normoxia

Hemodynamic measurements
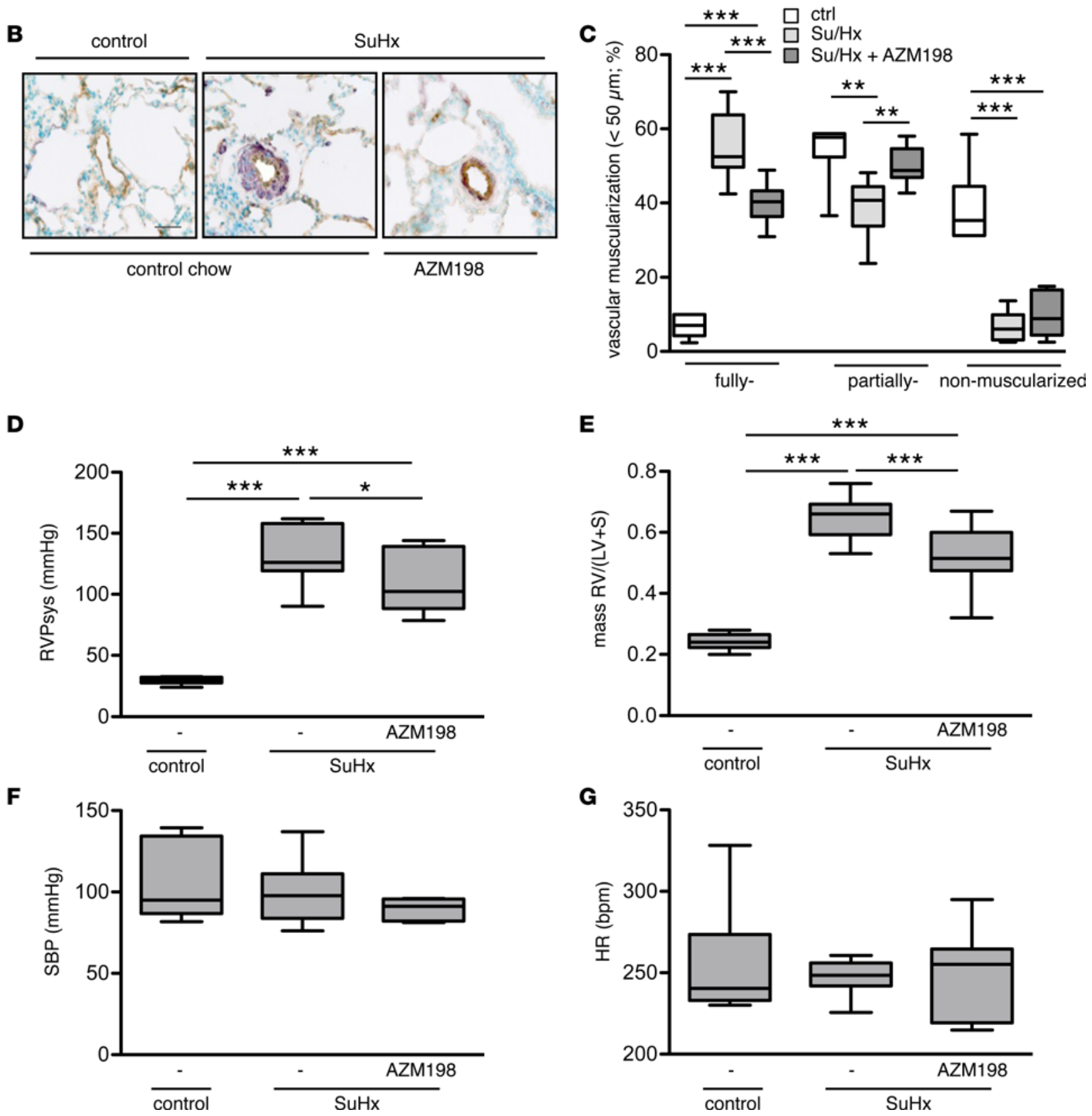

Figure 7. Effects of myeloperoxidase inhibition on pulmonary arterial hypertension induced by Sugen5416/hypoxia. Inhibition of myeloperoxidase (MPO) activity by AZM198 attenuates pulmonary hypertension and vascular remodeling in rats. (A) Schematic diagram illustrating the experimental design of the Sugen5416 (SU5416)/hypoxia (SuHx) model. (B) Representative images of lung sections (brown, von Willebrand factor; dark blue, $\alpha$-smooth muscle actin) demonstrating the muscularization of peripheral pulmonary arteries in control animals or animals with SuHx-induced pulmonary hypertension fed with control chow or AZM198-containing chow. Scale bar: $30 \mu \mathrm{m}$. (C) Vascular muscularization of small pulmonary arteries $(<50 \mu \mathrm{m})$ as expressed by the percentage of fully, partially, and nonmuscularized vessels (at least 80 were analyzed per animal). $n=6$ (ctrl), 9 (SuHx ctrl), 9 (SuHx AZM198); ${ }^{* *} P<0.01 ;{ }^{* * *} P<0.001$. (D) Right ventricular systolic pressure (RVPsys) (mmHg); $n=6$ (ctrl), 6 (SuHx ctrl), 10(SuHx AZM198). (E) Right ventricular (RV) hypertrophy expressed as RV/ $(\mathrm{LV}+\mathrm{S})$ ratio; $n=6$ (ctrl), 10 (SuHx ctrl), 14 (SuHx AZM198). (F) Systemic blood pressure (SBP) (mmHg) and (G) heart rate (HR) (bpm); $n=6$ (ctrl), 6 (SuHx ctrl), 9 (SuHx AZM198). All data are presented as median with interquartile range, whiskers indicate minimum to maximum. Statistical data assessed by 1-way ANOVA followed by LSD post hoc test. 


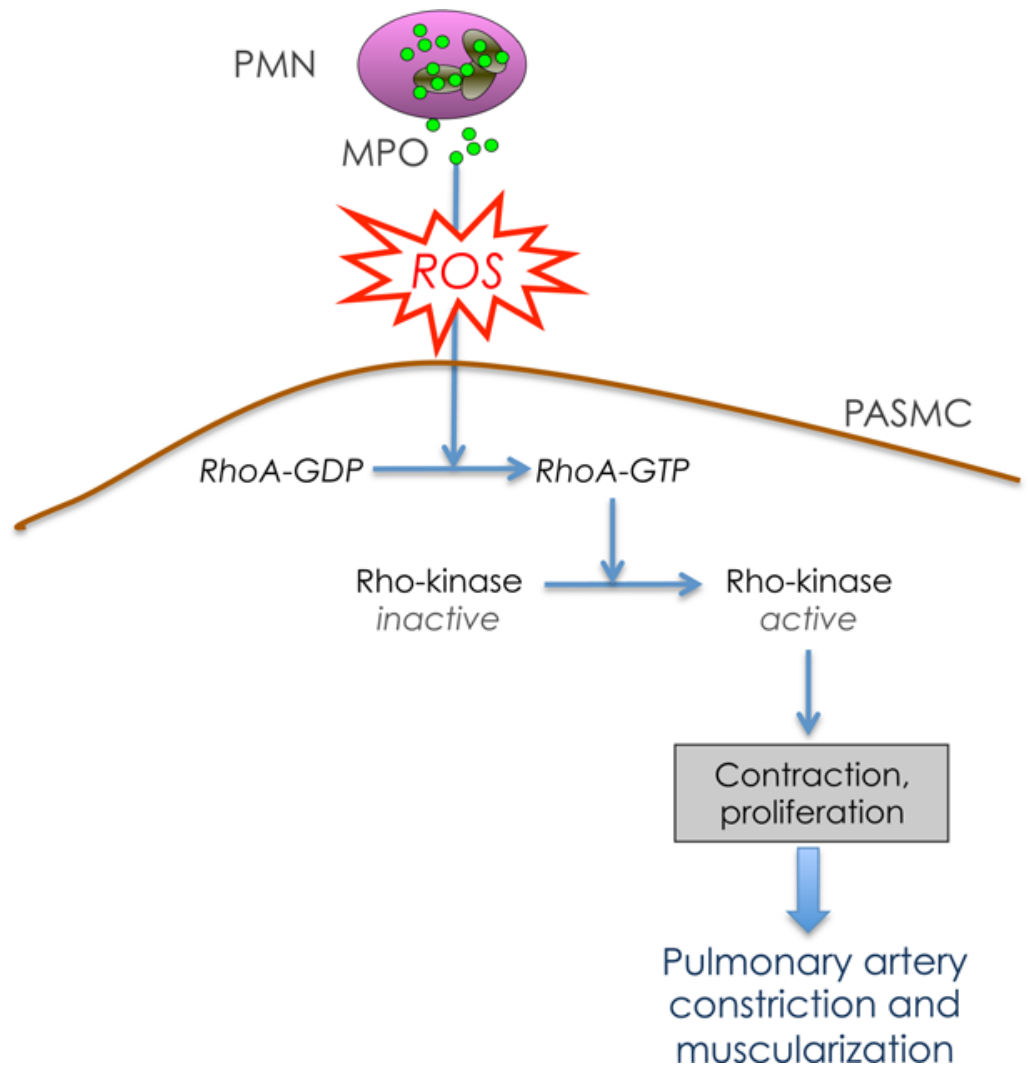

Figure 8. The role of myeloperoxidase in pulmonary arterial hypertension. Schematic illustrating the impact of myeloperoxidase (MPO) on pulmonary arterial constriction and muscularization via activation of the RhoA/Rho-kinase pathway. PMN, polymorphonuclear neutrophil; PASMC, pulmonary artery smooth muscle cell.

Clearly one must be cautious to transfer these findings directly to human $\mathrm{PAH}$, as all employed animal models exhibit important differences compared with human pathology (2). While our clinical data support a role of MPO in human PAH, they are observational and thus do not allow mechanistic insights. However, by including a relatively large number of purely Nice group $1 \mathrm{PAH}$ patients and by providing consistent findings in 2 independent cohorts, they are an important adjunct of the obtained experimental data. Still, only a controlled trial testing the effectiveness of pharmacological MPO inhibition in PAH patients would provide definite clarification regarding the pathophysiological relevance of MPO.

In conclusion, the data demonstrate an unprecedented mechanism of neutrophil-derived vascular dysfunctional and structural remodeling in the pulmonary circulation, yielding pulmonary hypertension. In light of the dire prognostic impact of PAH and the still-insufficient treatment options, MPO emerges not only as a critical mediator, but also as a potential therapeutic target in this disease.

\section{Methods}

Chemicals. All chemicals were purchased from MilliporeSigma, unless otherwise indicated.

Patients with PAH. Patients with PAH confirmed by right heart catheterization and age-matched control subject (individuals suffering from connective tissue disease without pulmonary hypertension or healthy volunteers) were investigated at 2 different centers (Cleveland Clinic and University Hospital Eppendorf, Hamburg, Germany). Patients with pulmonary hypertension in WHO diagnostic category $1(\mathrm{PAH})$ were included. Exclusion criteria were a confirmed or suspected infection and unstable coronary artery disease defined by clinical history, stress testing, and/or left heart catheterization. Diagnostic testing and therapeutic decisions were left entirely up to the treating clinician. A 6-minute walk test and right heart catheterization (mean PA pressures, pulmonary capillary wedge pressure, cardiac index) was performed at baseline. All-cause mortality since the date of blood sampling was assessed as the clinical outcome of interest and was ascertained by review of medical records. Data were collected in both a retrospective and prospective manner. MPO plasma levels were determined at baseline using CardioMPO ELISA (Cleveland Heart Lab, Cleveland, Ohio, USA). Elastase plasma levels were determined using ELISA (Immuno Biological Laboratories) and N-terminal (NT)-proBNP plasma levels were determined using the Elecsys method from Roche Diagnostics. To define cut-offs for elevation 
of MPO and NT-proBNP plasma levels, a ROC curve-based approach was chosen using death as the state variable and MPO or NT-proBNP plasma levels as the test variables. The respective value with the highest cumulative sensitivity and specificity was chosen as cut-off.

Human lung tissue samples. Paraffin-embedded samples of $4 \mathrm{PH}$ patients that underwent lung transplantation and 4 control patients that underwent lung cancer surgery at the University Medical Center Hamburg-Eppendorf were obtained from the Department of Pathology. Immediately after surgical resection, all specimens were fixed in formalin before ethanol dehydration and paraffin embedding.

Control samples were taken from tumor-, inflammation-, and fibrosis-free areas of lobectomy or pneumectomy specimens as determined by the attending pathologist.

Due to anonymization of samples before processing, the need for written consent was waived in accordance with national law (Hamburgisches Krankenhausgesetz, HmbKHG paragraph 12a, passage 1-5).

Mice and experimental design. Male C57B1/6J (WT) and MPO_tm1lus ( $\mathrm{Mpo}^{-/-}$, stock no. 4265) mice (The Jackson Laboratory) aged 9-12 weeks were maintained under constant normoxic $\left(21 \% \mathrm{O}_{2}\right)$ or hypoxic $(10$ $\% \mathrm{O}_{2}$ ) conditions for 2 hours, 4 hours, 7 days, or 28 days. An inhibitor to Rho-kinase (Y-27632, $10 \mathrm{mg} / \mathrm{kg}$ body weight [BW], Tocris Bioscience) was injected i.p. 1 hour prior to sacrifice or infused s.c. over 7 days via osmotic minipumps (1007D, Alzet), where indicated.

Determination of right ventricular pressure and volume and tissue preparation in mice. We anesthetized mice with isoflurane (Abbott), administered low-dose buprenorphin (Essex-Pharma; $0.05 \mathrm{mg} / \mathrm{kg} \mathrm{BW} \mathrm{s.c.)} \mathrm{for} \mathrm{analgesia,}$ and placed them on a heating pad to maintain body temperature. Following endotrachial intubation, animals were ventilated with 150 strokes/min and stroke volume of $7 \mu \mathrm{l} / \mathrm{g} \mathrm{BW}$. We cannulated the left jugular vein with a PE-10 tubing and a solution of $12.5 \%$ BSA (MilliporeSigma, $2 \mu 1 / \mathrm{g} \mathrm{BW}$ ) was infused. A microtip conductance pressure-volume catheter (1 F, PVR-1035 NR, Millar Instruments) was inserted via the right jugular vein into the right ventricle. Heart rate was maintained between $400-500 \mathrm{bpm}$ by adjusting the concentration of isoflurane accordingly. Right ventricular pressure and volume was recorded repeated times while ventilation was discontinued for 2 seconds with an ADInstruments PowerLab 8/30 system (ADInstruments). Volume calibration was performed using ADInstruments volume calibration cuvette. Right ventricular systolic pressure was evaluated and $\mathrm{E}_{\mathrm{a}}$ was calculated dividing end- systolic pressure by stroke volume $\left(\mathrm{P}_{\mathrm{es}} / \mathrm{SV}\right)$.

After completion of hemodynamic studies, the thoracic cavity was opened, blood was drawn from the caval vein, lungs and hearts were flushed with saline via the jugular vein, and hearts and lungs were excised. For ventricular weight measurement, atria were removed, the free right ventricular wall was dissected, and each ventricle was weighed. The ratio of $\mathrm{RV}$ to left ventricle plus septum $(\mathrm{RV} /[\mathrm{LV}+\mathrm{S}])$ was used as an index of right ventricular hypertrophy.

Heparinized blood was centrifuged, and plasma was stored on $-80^{\circ} \mathrm{C}$ until further analysis. MPO plasma levels were determined with MPO ELISA (Hycult Biotech) according to manufacturer's instructions.

For WBC counts, blood was anticoagulated with EDTA and stained with Turk's solution (1:20). Afterward, leukocytes were counted under the microscope in a Neubauer improved cell counting chamber.

I.v. MPO infusion experiments. Male C57B1/6J (WT) and $\mathrm{MPO}$ tm1lus $\left(\mathrm{Mpo}^{-/}\right)$mice aged 9-12 weeks were anesthetized with isoflurane (Abbott) and received buprenorphin (Essex-Pharma) for analgesia. An osmotic minipump (1007D, Alzet) filled with recombinant MPO (R\&D Systems) and connected to an Alzet mouse jugular catheter (polyurethane, OD $0.36 \mathrm{~mm}$ ) was implanted s.c. The catheter was inserted to the left jugular vein and fixed with suture and tissue adhesive. MPO was continuously infused over 7 days with a dose of $0.05 \mathrm{ng} / \mathrm{g} / \mathrm{min}$.

Isometric force measurements. We anesthetized mice with isoflurane as described and following thoracotomy-explanted sections of the PAs, which were immediately kept in Krebs-Henseleit buffer on ice. We cut 2-mm segments and mounted them onto steel wires connected to force transducers of a standard organ bath filled with $25 \mathrm{ml} \mathrm{Krebs-Henseleit} \mathrm{buffer} \mathrm{at} 37^{\circ} \mathrm{C}$ and gassed with carbogen. Each ring was equilibrated for 30 minutes and continuously stretched up to a tension of $0.7 \mathrm{~g} . \mathrm{KCl}(80 \mathrm{mM})$ was added twice to each bath with subsequent washing. To create a dose-response curve to $\mathrm{PGF}_{2} \alpha$, we added it to the bath with concentration (in $\mu \mathrm{M}$ ) of $0.1,0.2,0.4,1,2,4$, and 10 . Thereafter, a dose response to ACh was recorded with ACh concentrations from $1 \times 10^{-9} \mathrm{M}$ in steps of half $\log _{10}$ levels up to a concentration of $1 \times 10^{-5.5} \mathrm{M}$.

SuHx-induced pulmonary hypertension in rats. Male Sprague Dawley rats (8 weeks old, Charles River Laboratories) were s.c. injected with Sugen 5416 (20 mg/kg dissolved in DMSO) and exposed to a $10 \%$ normobaric oxygen hypoxia for 3 weeks (Biospherix hypoxia chamber), followed by exposure to normoxia for another 2 weeks. The rats were fed control chow (R70 chow, Lantmännen) or chow containing the MPO 
inhibitor AZM198 (synthesized by AstraZeneca) at a concentration estimated to yield a daily dose of 500 $\mu \mathrm{mol} / \mathrm{kg}$ and a total plasma concentration of at least $2 \mu \mathrm{mol} / 1$ (35). Adequate exposure was confirmed by quantifying the plasma AZM198 concentration upon termination of the rats (data not shown).

Determination of right ventricular pressure and tissue preparation in rats. Hemodynamic analysis was conducted after 35 days. Rats were anesthetized with a premixed combination of $100 \mathrm{mg} / \mathrm{kg}$ ketamine and $4 \mathrm{mg} /$ $\mathrm{kg}$ xylazine, tracheostomized, and artificially ventilated. Right ventricular systolic pressure was determined utilizing a fluid-filled catheter, inserted into the right ventricle via the jugular vein. SBP was monitored in the carotid artery using a Millar pressure catheter (SPR-320). The catheter information was amplified by a PowerLab amplifier and converted to pressure curves using LabChart7 software (AD instruments).

Following hemodynamic measurements, the heart was removed and RV hypertrophy was assessed as described above.

Histology and pulmonary vascular morphometry. Lung tissue preparation, sectioning, staining, and vascular morphometry were done as described (36). Lungs were perfused with chilled PBS for 10 minutes, extracted, and fixed in 4\% paraformaldehyde. Following dehydration, lungs were embedded in paraffin and cut into $3-\mu \mathrm{m}$ sections. The degree of muscularization of peripheral PAs was assessed by double-immunostaining the sections with an anti- $\alpha$-smooth muscle actin antibody (dilution 1:900, clone 1A4, catalog A2547, MilliporeSigma) and anti-von Willebrand factor antibody (vWF, dilution 1:900, polyclonal, cata$\log$ A0082, Dako). In each animal, 80-100 intraacinar arteries at a size between 20-150 $\mu$ m accompanying either alveolar ducts or alveoli were analyzed using a computerized morphometric system (QWin; Leica) and categorized as nonmuscularized (i.e., no apparent muscle), partially muscularized (i.e., with only a crescent of muscle), or fully muscularized (i.e., with a complete medial coat of muscle). In all cases, investigators and slides were blinded for the genotype or treatment group of the respective animals.

MPO immunoreactivity. Paraffin-embedded human lung specimens were cut to $3-\mu \mathrm{m}$ sections and treated with a polyclonal antibody to MPO (rabbit, 1:100; catalog 475915, Merck). Histofine Simple Stain (Nichirei Biosciences) was used as a secondary antibody, and sections were treated with 3-amino 9-ethyl carbazole (AEC) solution to allow for development of a brown precipitate. Tissue was counterstained with hematoxyline. Images were acquired using a Keyence BZ-9000 microscope.

$P M N$ infiltration. Mice were anesthetized with isoflurane as described, and lungs were flushed with PBS via the jugular vein, excised, frozen in OCT compound, and cut to $4-\mu \mathrm{m}$ sections. Frozen lung specimens were fixed with acetone. Sections were incubated with rat anti-mouse neutrophil Ly6G primary antibody (1:40, clone NIMP-R14, catalog HM1039, Hycult Biotech), and endogenous peroxidase activity was blocked. Secondary antibody was HRP-labeled rabbit anti-rat (1:100, catalog p0450, polyclonal, Dako), and tertiary antibody was HRP-labeled goat anti-rabbit (1:500, catalog PI-1000, polyclonal, Vectorlabs) in 3\% normal mouse serum. PMN were stained with $\mathrm{AEC}$ solution, and tissue was counterstained with hematoxyline. Images were acquired using a Prosilica GC camera (Allied Vision Technologies) mounted on a Leica DMLB light microscope.

Western blot. Lung specimens were lysed in lysis buffer (20 mM Tris- $\mathrm{HCl}$ [pH 7.5], $250 \mathrm{mM}$ sucrose, $20 \mathrm{mM}$ EDTA, 3 mM EGTA, and 0.1\% Triton X-100, supplemented with 10× EDTA-free Protease Inhibitor Tablets and 10× PhoSTOP; Roche Diagnostics) using the Tissue Lyzer (Qiagen). Homogenates were centrifuged at $14,000 \mathrm{~g}\left(4^{\circ} \mathrm{C}, 10\right.$ minutes $)$, and the supernatant was recovered. Proteins were separated by SDS-PAGE and transferred to nitrocellulose membranes. After blocking with either 5\% nonfat milk or 5\% BSA in TBST (20 mM Tris- $\mathrm{HCl}$ [pH 7.5], $137 \mathrm{mM} \mathrm{NaCl}, 0.1 \%$ [v/v] Tween 20), membranes were incubated with primary antibodies to phospho-MYPT (Thr-696, 1:2,000, polyclonal, catalog ABS45, MilliporeSigma), VASP (1:2,000, clone IG731, catalog 0012-02, ImmunoGlobe) (detecting Ser-157 dephospho and Ser-157 phospho VASP, values are expressed as Ser-157 phospho VASP related to dephospho VASP, reflecting cGMP-dependent protein kinase activity), iNOS (1:250, IgG2a, catalog 610431, BD Biosciences), or GAPDH (1:5,000, clone 14C10, catalog 2118, Cell Signaling Technology), followed by HRP-conjugated secondary antibodies (1:10,000, Vector Laboratories); chemiluminescence signals were detected on film and analyzed densitometrically with ImageJ.

Cytokine immunoassay. Mice were anesthetized with isoflurane as described, and lungs were flushed with PBS via the jugular vein and excised. Lung homogenates were prepared as described for Western blot analysis and were analyzed for cytokines by using a LEGENDplex Mouse Inflammation Panel (13-plex) (BioLegend) according to manufacturer's instructions.

Quantitative PCR. For quantitative mRNA expression analyses, lung tissue was collected. RNA was isolated using an RNA isolation kit (RNeasy Mini Kit) according to manufacturer's instruction. First-strand synthesis with a High Capacity cDNA Reverse Transcription Kit (Applied Biosystems) was performed 
Table 2. Primers

\begin{tabular}{|c|c|c|}
\hline Inos & forward & 5'-CTC ACT GGG ACA GCA CAG AA-3' \\
\hline reverse & 5'-GGT CAA ACT CTT GGG GTT CA-3' & \\
\hline Ccr2 & forward & 5'-CCT GCA AAG ACC AGA AGA GG-3' \\
\hline Ccl5 & forward & 5'-GTC CCC ACG TCA AGG AGT AT-3' \\
\hline reverse & 5'-CCC ACT TCT TCT СTC GGT TG-3' & \\
\hline 185 & forward & 5'-GTA ACC CGT TGA ACC CCA TT-3' \\
\hline reverse & 5'-TCT GCA TCG TTA СTT TGG GCC-3' & \\
\hline $1123 a$ & forward & 5'-GCA СCT GCT TCA CTC TGA CA-3' \\
\hline reverse & 5'-TAG AAC TCA GGC TGG GCA TC-3' & \\
\hline Tnf & forward & 5'-CCC CAA AGG GAT GAG AAG TT-3' \\
\hline reverse & 5'-CAC TTG GTG GTT TCC TAC GA-3' & \\
\hline Cxc/10 & forward & 5'-TCC TTC TCC TCC CTA GCT CA-3' \\
\hline reverse & 5'-TCT TCC ATA TAC AAT GCA AAG CA-3' & \\
\hline
\end{tabular}

according to manufacturer's instructions and quantitative PCR (qPCR) was performed with a SsoFast EvaGreen Supermix with Low ROX (Bio-Rad) at an annealing temperature of $60^{\circ} \mathrm{C}$ in a $\mathrm{C} 1000$ Touch Thermal Cycler (CFX96 Real-Time System, Bio-Rad). Target gene mRNA expression was normalized to mRNA expression of $18 \mathrm{~s}$ by $\Delta \Delta \mathrm{CT}$ method (Table 2).

A TaqMan Gene Expression Assay was used for Rock1 (Mm00485745_m1) and Rock2 (Mm01270843_ m1) (Applied Biosystems).

Cell culture. Human PASMCs were purchased from Lonza and cultured in SmGM media containing $5 \% \mathrm{FBS}$ (Invitrogen) in $5 \% \mathrm{CO}_{2}$ at $37^{\circ} \mathrm{C}$. All experiments were performed using PASMCs from passage 3-7. PASMCs were grown to $70 \%-80 \%$ confluency in 6-well plates. Cells were serum starved for 24 hours prior to cell treatments; medium was supplemented with $0.025 \%$ BSA during that time. Active human MPO $(0.1$ or $1 \mu \mathrm{g} / \mathrm{ml}$, Planta Natural Products) with hydrogen peroxide (0.4 or $4 \mu \mathrm{M}$, respectively), Q91T MPO (1 $\mu \mathrm{g} / \mathrm{ml}$, provided by P.G. Furtmüller (University of Life Sciences and Natural Resources, Vienna, Austria), or $10 \%$ FCS was added in basal medium without serum. After 30 seconds, supernatants were removed and cells were washed in ice cold PBS. For analysis of RhoA, activity cells were lysed and processed with the G-LISA RhoA activation assay (Cytoskeleton Inc.) according to the manufacturer's recommendation. For analysis of Rho-kinase activity, cell supernatants were removed, and PASMCs were snap-frozen in culture dishes, thawed in ice cold lysis buffer (20 mM Tris- $\mathrm{HCl}$ [pH 7.5], $250 \mathrm{mM}$ sucrose, $20 \mathrm{mM}$ EDTA, $3 \mathrm{mM}$ EGTA, and $0.5 \%$ Triton X-100, supplemented with 10× EDTA-free Protease Inhibitor cocktail and 10× PhoSTOP; Roche Diagnostics), and processed as described for Western blot.

Statistics. Continuous variables were tested for normal distribution by using the Kolmogorov-Smirnoff test. Statistical analysis was performed by 1-way ANOVA followed by Bonferroni or LSD post hoc tests, $\chi^{2}$ test, 2-tailed Student's $t$ test, or Wilcoxon rank sum test, as appropriate. Correlations were computed using Spearman's nonparametric test. Survival analyses were performed using Kaplan-Meier estimates and log-rank tests. Patients were divided into 2 groups with low and high MPO plasma levels based on a ROC curve analysis using death as the state variable and MPO plasma levels as the test variable. A value of $P<$ 0.05 was considered statistically significant. Calculations were carried out using SPSS Statistics 20 for Mac.

Study approval. The patient study was approved by the IRBs of the Cleveland Clinic and the University Hospital Eppendorf (Hamburg, Germany), and written informed consent was obtained from every patient. The study was performed in accordance with the declaration of Helsinki.

All animal studies were approved by the University of Hamburg and University of Cologne Animal Care and Use Committees and complied with the national guidelines for the care and use of laboratory animals. 


\section{Author contributions}

AK designed the experimental portion of the project, performed experiments and statistical analysis, and prepared the manuscript. EB performed the SuHx experiments and statistical analysis, and wrote SuHx portion of the manuscript. KF and TKR in part designed the project and performed experiments. DL, LR, MB, CK, TR, M. Adam, DM, MM, KM, and LK performed experiments. EM provided AZM198 and participated in design of SuHx experimental protocol and data analysis. SM was responsible for the patient cohort of Hamburg, Germany. GAH, M. Aytekin, and RAD were responsible for the patient cohort of Cleveland, Ohio, USA. JKH and HK were responsible for the cohort of Hamburg, for lung specimens, and for making suggestions on the project. SR and SLH provided suggestions on the project and revised the manuscript. RTS was responsible for analysis of structural remodeling in mouse lung tissue. SB supervised the project. VR supervised the project and wrote the manuscript.

\section{Acknowledgments}

We wish to thank Hartwig Wieboldt, Lucia Bino, and Silvia Kremserova for expert technical assistance; Julia Müller for providing lung tissue samples of PAH and lung cancer patients; and Paul-Georg Furtmüller for providing Q91T MPO. This work was supported by the Deutsche Forschungsgemeinschaft DFG [KL 2516/1-1 to A.K.; HE 6855/1-1 to J.K.H.; BA 1870/9-1 and BA 1870/10-1 to S.B.; RU 1876/11 and RU 1876/3-1 to V.R.]; the Center for Molecular Medicine Cologne (CMMC) [project 2-GA to A.K., S.B. and V.R. and 3-RP to M.M., S.B. and V.R.] and by MEYS CR [project no. LQ1605 from the NPU to A.K and L.K.]. G.A.H. was supported by the Gilead Sciences Research Scholars Program Award in Pulmonary Arterial Hypertension. The study was supported in part by National Institutes of Health grants [P01HL076491, P01HL098055, P01HL103453 and HL113452 to S.L.H.]. S.L.H. is also partially supported by a gift from the Leonard Krieger fund.

Address correspondence to: Volker Rudolph or Anna Klinke, Heart Center, University of Cologne, Kerpener Strasse 62, 50937 Cologne, Germany. Phone: 49.221.47832412; Email: volker.rudolph@uk-koeln.de (V. Rudolph). Phone: 49.221.47887402; Email: anna.klinke@uk-koeln.de (A. Klinke).

1. Simonneau G, et al. Updated clinical classification of pulmonary hypertension. J Am Coll Cardiol. 2013;62(25 Suppl):D34-D41.

2. Stenmark KR, Meyrick B, Galie N, Mooi WJ, McMurtry IF. Animal models of pulmonary arterial hypertension: the hope for etiological discovery and pharmacological cure. Am J Physiol Lung Cell Mol Physiol. 2009;297(6):L1013-L1032.

3. O'Callaghan DS, et al. Treatment of pulmonary arterial hypertension with targeted therapies. Nat Rev Cardiol. 2011;8(9):526-538.

4. Price LC, et al. Inflammation in pulmonary arterial hypertension. Chest. 2012;141(1):210-221.

5. Savai R, et al. Immune and inflammatory cell involvement in the pathology of idiopathic pulmonary arterial hypertension. $A m$ J Respir Crit Care Med. 2012;186(9):897-908.

6. Lanza F. Clinical manifestation of myeloperoxidase deficiency. J Mol Med. 1998;76(10):676-681.

7. Exner M, et al. Myeloperoxidase predicts progression of carotid stenosis in states of low high-density lipoprotein cholesterol. J Am Coll Cardiol. 2006;47(11):2212-2218.

8. Brevetti G, et al. Myeloperoxidase, but not C-reactive protein, predicts cardiovascular risk in peripheral arterial disease. Eur Heart J. 2008;29(2):224-230.

9. Baldus S, et al. Myeloperoxidase serum levels predict risk in patients with acute coronary syndromes. Circulation. 2003;108(12):1440-1445.

10. Brennan ML, et al. Prognostic value of myeloperoxidase in patients with chest pain. N Engl J Med. 2003;349(17):1595-1604.

11. Lau D, Baldus S. Myeloperoxidase and its contributory role in inflammatory vascular disease. Pharmacol Ther. 2006;111(1):16-26.

12. Eiserich JP, et al. Myeloperoxidase, a leukocyte-derived vascular NO oxidase. Science. 2002;296(5577):2391-2394.

13. Baldus S, et al. Endothelial transcytosis of myeloperoxidase confers specificity to vascular ECM proteins as targets of tyrosine nitration. J Clin Invest. 2001;108(12):1759-1770.

14. Eiserich JP, et al. Formation of nitric oxide-derived inflammatory oxidants by myeloperoxidase in neutrophils. Nature. 1998;391(6665):393-397.

15. Zhang C, et al. L-arginine chlorination products inhibit endothelial nitric oxide production. J Biol Chem. 2001;276(29):27159-27165.

16. von Leitner EC, et al. Pathogenic cycle between the endogenous nitric oxide synthase inhibitor asymmetrical dimethylarginine and the leukocyte-derived hemoprotein myeloperoxidase. Circulation. 2011;124(24):2735-2745.

17. Rudolph TK, et al. Myeloperoxidase deficiency preserves vasomotor function in humans. Eur Heart J. 2012;33(13):1625-1634

18. Morimont P, et al. Effective arterial elastance as an index of pulmonary vascular load. Am J Physiol Heart Circ Physiol. 2008;294(6):H2736-H2742.

19. Wauthy P, Abdel Kafi S, Mooi WJ, Naeije R, Brimioulle S. Inhaled nitric oxide versus prostacyclin in chronic shunt-induced pulmonary hypertension. J Thorac Cardiovasc Surg. 2003;126(5):1434-1441

20. Knock GA, Ward JP. Redox regulation of protein kinases as a modulator of vascular function. Antioxid Redox Signal. 2011;15(6):1531-1547. 
21. Rudolph V, et al. Myeloperoxidase acts as a profibrotic mediator of atrial fibrillation. Nat Med. 2010;16(4):470-474.

22. Oka M, et al. Rho kinase-mediated vasoconstriction is important in severe occlusive pulmonary arterial hypertension in rats. Circ Res. 2007;100(6):923-929.

23. Taraseviciene-Stewart L, et al. Inhibition of the VEGF receptor 2 combined with chronic hypoxia causes cell death-dependent pulmonary endothelial cell proliferation and severe pulmonary hypertension. FASEB J. 2001;15(2):427-438.

24. Undurti A, Huang Y, Lupica JA, Smith JD, DiDonato JA, Hazen SL. Modification of high density lipoprotein by myeloperoxidase generates a pro-inflammatory particle. J Biol Chem. 2009;284(45):30825-30835.

25. Klinke A, et al. Myeloperoxidase attracts neutrophils by physical forces. Blood. 2011;117(4):1350-1358.

26. Fagan KA, et al. Upregulation of nitric oxide synthase in mice with severe hypoxia-induced pulmonary hypertension. Respir Res. 2001;2(5):306-313.

27. Le Cras TD, Xue C, Rengasamy A, Johns RA. Chronic hypoxia upregulates endothelial and inducible NO synthase gene and protein expression in rat lung. Am J Physiol. 1996;270(1 Pt 1):L164-L170.

28. Gosgnach W, Messika-Zeitoun D, Gonzalez W, Philipe M, Michel JB. Shear stress induces iNOS expression in cultured smooth muscle cells: role of oxidative stress. Am J Physiol, Cell Physiol. 2000;279(6):C1880-C1888.

29. Kristof AS, Marks-Konczalik J, Moss J. Mitogen-activated protein kinases mediate activator protein-1-dependent human inducible nitric-oxide synthase promoter activation. J Biol Chem. 2001;276(11):8445-8452.

30. Schermuly RT, Ghofrani HA, Wilkins MR, Grimminger F. Mechanisms of disease: pulmonary arterial hypertension. Nat Rev Cardiol. 2011;8(8):443-455.

31. Nossaman BD, Kadowitz PJ. The role of the RhoA/rho-kinase pathway in pulmonary hypertension. Curr Drug Discov Technol. 2009;6(1):59-71.

32. Shibata R, et al. Role of Rho-associated kinase in neointima formation after vascular injury. Circulation. 2001;103(2):284-289.

33. Seasholtz TM, Majumdar M, Kaplan DD, Brown JH. Rho and Rho kinase mediate thrombin-stimulated vascular smooth muscle cell DNA synthesis and migration. Circ Res. 1999;84(10):1186-1193.

34. Sommer N, et al. Regulation of hypoxic pulmonary vasoconstriction: basic mechanisms. Eur Respir J. 2008;32(6):1639-1651.

35. Björnsdottir $\mathrm{H}$, et al. Neutrophil NET formation is regulated from the inside by myeloperoxidase-processed reactive oxygen species. Free Radic Biol Med. 2015;89:1024-1035.

36. Schermuly RT, et al. Reversal of experimental pulmonary hypertension by PDGF inhibition. J Clin Invest. 2005;115(10):2811-2821. 